\title{
Auger electron angular distributions following excitation or ionization of the I $3 d$ level in methyl iodide
}

Ruaridh Forbes, Alberto De Fanis, Cédric Bomme, Daniel Rolles, Stephen T. Pratt, Ivan Powis, Nicholas A. Besley, Saikat Nandi, Aleksandar R. Milosavljević, Christophe Nicolas, John D. Bozek, Jonathan G. Underwood, and David M. P. Holland

Citation: J. Chem. Phys. 149, 094304 (2018); doi: 10.1063/1.5045640

View online: https://doi.org/10.1063/1.5045640

View Table of Contents: http://aip.scitation.org/toc/jcp/149/9

Published by the American Institute of Physics

\section{Articles you may be interested in}

Photoionization of the iodine $3 \mathrm{~d}, 4 \mathrm{~s}$, and $4 \mathrm{p}$ orbitals in methyl iodide

The Journal of Chemical Physics 149, 144302 (2018); 10.1063/1.5035496

Coulomb explosion imaging of $\mathrm{CH}_{3} \mathrm{l}$ and $\mathrm{CH}_{2} \mathrm{Cll}$ photodissociation dynamics

The Journal of Chemical Physics 149, 204313 (2018); 10.1063/1.5041381

Time-resolved multi-mass ion imaging: Femtosecond UV-VUV pump-probe spectroscopy with the PImMS camera

The Journal of Chemical Physics 147, 013911 (2017); 10.1063/1.4978923

Excited state wavepacket dynamics in $\mathrm{NO}_{2}$ probed by strong-field ionization

The Journal of Chemical Physics 147, 054305 (2017); 10.1063/1.4996461

Excited state non-adiabatic dynamics of the smallest polyene, trans 1,3-butadiene. I. Time-resolved photoelectron-photoion coincidence spectroscopy

The Journal of Chemical Physics 148, 164302 (2018); 10.1063/1.5016452

An experimental and theoretical study of the photoelectron spectra of cis-dichloroethene: Valence shell vertical ionization and vibronic coupling in the low-lying cationic states

The Journal of Chemical Physics 149, 074306 (2018); 10.1063/1.5033425

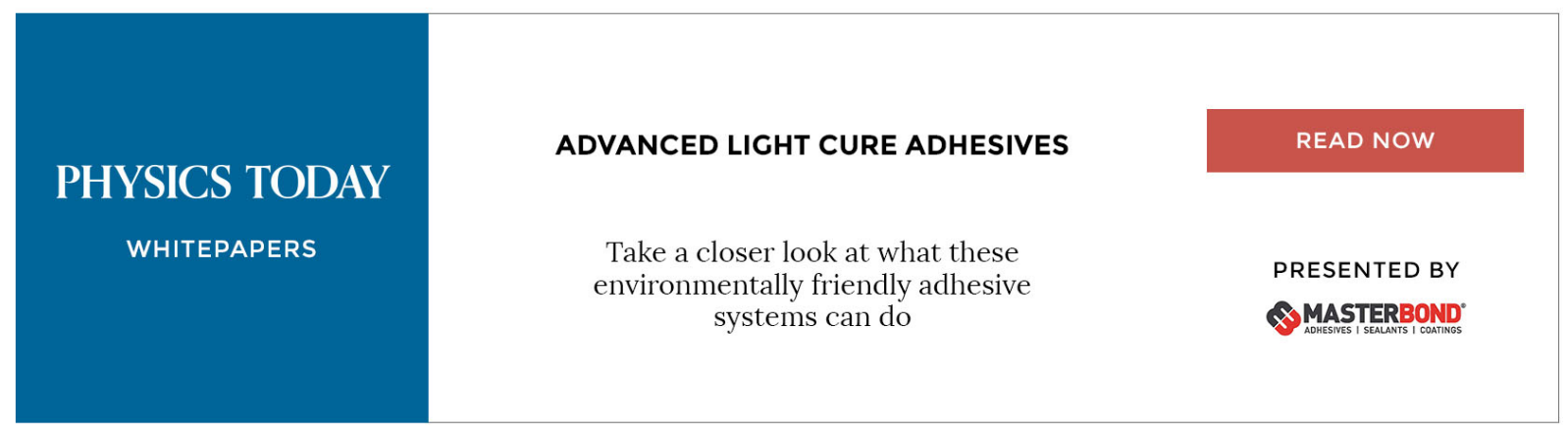




\title{
Auger electron angular distributions following excitation or ionization of the I $3 d$ level in methyl iodide
}

\author{
Ruaridh Forbes, ${ }^{1,2}$ Alberto De Fanis, ${ }^{3}$ Cédric Bomme, ${ }^{4}$ Daniel Rolles, ${ }^{5}$ \\ Stephen T. Pratt, ${ }^{6}$ Ivan Powis, ${ }^{7}$ Nicholas A. Besley, ${ }^{7}$ Saikat Nandi, ${ }^{8}$ \\ Aleksandar R. Milosavljević, ${ }^{8}$ Christophe Nicolas, ${ }^{8}$ John D. Bozek, ${ }^{8}$ \\ Jonathan G. Underwood, ${ }^{1}$ and David M. P. Holland ${ }^{9, a)}$ \\ ${ }^{1}$ Department of Physics and Astronomy, University College London, Gower Street, London \\ WC1E 6BT, United Kingdom \\ ${ }^{2}$ Department of Physics, University of Ottawa, 150 Louis Pasteur, Ottawa, Ontario K1N 6N5, Canada \\ ${ }^{3}$ European XFEL, GmbH, Holzkoppel 14, 22869 Schenefeld, Germany \\ ${ }^{4}$ Deutsches Elektronen-Synchrotron (DESY), 22607 Hamburg, Germany \\ ${ }^{5}$ J. R. Macdonald Laboratory, Department of Physics, Kansas State University, Manhattan, Kansas 66506, USA \\ ${ }^{6}$ Chemical Sciences and Engineering Division, Argonne National Laboratory, Argonne, Illinois 60439, USA \\ ${ }^{7}$ School of Chemistry, University of Nottingham, Nottingham NG7 2RD, United Kingdom \\ ${ }^{8}$ Synchrotron SOLEIL, l'Orme des Merisiers, Saint-Aubin, BP 48, 91192 Gif-sur-Yvette, France \\ ${ }^{9}$ Daresbury Laboratory, Daresbury, Warrington, Cheshire WA4 4AD, United Kingdom
}

(Received 22 June 2018; accepted 14 August 2018; published online 6 September 2018)

\begin{abstract}
Auger electron spectra following excitation or ionization of the I $3 \mathrm{~d}$ level in $\mathrm{CH}_{3} \mathrm{I}$ have been recorded with horizontally or vertically plane polarized synchrotron radiation. These spectra have enabled the Auger electron angular distributions, as characterized by the $\beta$ parameter, to be determined. The I $3 \mathrm{~d}$ photoionization partial cross section of $\mathrm{CH}_{3} \mathrm{I}$ has been calculated with the continuum multiple scattering approach, and the results show that in the photon energy range over which Auger spectra were measured, the I $3 \mathrm{~d}$ cross section exhibits an atomic-like behavior and is dominated by transitions into the $\varepsilon f$ continuum channel. In this limit, the theoretical value of the alignment parameter $\left(A_{20}\right)$ characterizing the core ionized state in an atom becomes constant, independent of photon energy. This theoretical value has been used to obtain the Auger electron intrinsic anisotropy parameters $\left(\alpha_{2}\right)$ from the $\beta$ parameters extracted from our normal (non-resonant) molecular Auger spectra. The resulting anisotropy parameters for the $\mathrm{M}_{45} \mathrm{~N}_{45} \mathrm{~N}_{45}$ transitions in $\mathrm{CH}_{3} \mathrm{I}$ have been compared to those calculated for the corresponding transitions in xenon, and the experimental and theoretical results are in good agreement. Anisotropy parameters have also been measured for the $\mathrm{M}_{45} \mathrm{~N}_{1} \mathrm{~N}_{45}$, $\mathrm{M}_{45} \mathrm{~N}_{23} \mathrm{~N}_{45}$, and $\mathrm{M}_{45} \mathrm{~N}_{45} \mathrm{O}_{23}$ transitions. For the $\mathrm{M}_{45} \mathrm{~N}_{1} \mathrm{~N}_{45}$ and $\mathrm{M}_{45} \mathrm{~N}_{23} \mathrm{~N}_{45}$ Auger decays in $\mathrm{CH}_{3} \mathrm{I}$, the experimentally derived angular distributions do not exhibit the strong dependence on the final ionic state that is predicted for these transitions in xenon. Resonantly excited Auger spectra have been recorded at 620.4 and $632.0 \mathrm{eV}$, coinciding with the $\mathrm{I}_{3} \mathrm{~d}_{5 / 2} \rightarrow \sigma^{*}$ and $3 \mathrm{~d}_{3 / 2} \rightarrow \sigma^{*}$ transitions, respectively. The resulting Auger electron angular distributions for the $M_{4} N_{45} N_{45}$ and $M_{5} N_{45} N_{45}$ decays were found to exhibit a higher anisotropy than those for the normal process. This is due to the larger photo-induced alignment in the neutral core excited state. For a particular Auger transition, the Auger electron kinetic energy measured in the resonantly excited spectrum is higher than that in the normal spectrum. This shift, due to the screening provided by the electron excited into the $\sigma^{*}$ orbital, has been rationalized by calculating orbital ionization energies of I $3 \mathrm{~d}$ excited and I $3 \mathrm{~d}$ ionized states in $\mathrm{CH}_{3}$ I. Published by AIP Publishing. https://doi.org/10.1063/1.5045640
\end{abstract}

\section{INTRODUCTION}

Conventional Auger electron spectroscopy, where a core ionized atom or molecule is created by an electron beam or a resonance line photon source, has yielded a wealth of information on electronic energy levels and lifetimes, and on the associated decay mechanisms. ${ }^{1,2}$ The subsequent use of synchrotron radiation as the ionization source, due to its

a)Electronic mail: david.holland@stfc.ac.uk tunability and polarization properties, has resulted in Auger spectroscopy becoming an increasingly sophisticated and versatile technique. ${ }^{3-8}$ To date, the majority of the experiments employing synchrotron radiation have been performed on molecules composed of low atomic number elements, especially carbon, nitrogen, and oxygen. Auger decay following core excitation or ionization in these systems involves valence electrons. When heavier atoms are present in the molecule, Auger decay can involve inner-shell, inner-valence, and outer-valence electrons, and the processes become far more complex. 
The tunability of synchrotron radiation has been exploited in the closely related technique of resonant Auger spectroscopy, ${ }^{3-8}$ where photoabsorption at an energy just below a core level ionization threshold initially leads to the formation of a neutral excited state lying far above the ground state. This intermediate state may decay through an Auger transition to produce a singly charged ion, with the kinetic energy of the ejected electron being determined by the difference between the neutral intermediate and the final ionic state energies.

One of the early investigations to take advantage of the continuous wavelength coverage afforded by synchrotron radiation concerned a study of the competition between Auger emission and molecular dissociation in core excited $\mathrm{HBr}^{9}$ Specifically, the photon energy was chosen to excite the $\mathrm{Br}$ $3 \mathrm{~d} \rightarrow \sigma^{*}$ transition, where $\sigma^{*}$ corresponds to a low energy antibonding orbital which is unoccupied in the neutral ground state. Although electronic relaxation is usually faster than dissociation, in this particular case, the time scale for dissociation is similar to that for Auger emission (a few fs). Thus, due to the fast dissociation of the core excited parent molecule, the electron spectrum was found to contain peaks associated with autoionizing atomic bromine. A subsequent study of the corresponding I $4 \mathrm{~d} \rightarrow \sigma^{*}$ transition in $\mathrm{CH}_{3} \mathrm{I}^{10}$ provided no evidence of atomic Auger decay. Instead, the photoelectron spectrum exhibited peaks due to molecular autoionization. These pioneering studies on $\mathrm{HBr}$ and $\mathrm{CH}_{3}$ I prompted extensive experimental and theoretical investigations on $\mathrm{HCl}$ and other closely related halogen containing molecules. In particular, the $\mathrm{Cl} 2 \mathrm{p} \rightarrow \sigma^{*}$ and $\mathrm{Cl} 2 \mathrm{p} \rightarrow$ Rydberg excitations were studied in detail. ${ }^{11-19}$ More recent studies have shown (Ref. 20 and references therein) that the competition between Auger decay and neutral dissociation is a more general phenomenon, but the rapid separation of atoms in the hydrogen halide systems enhances its effects.

While the tunability of synchrotron radiation has been used to advantage in many Auger electron studies, much less use has been made of its polarization properties. Plane polarized radiation allows the angular distributions of the Auger electrons to be measured, and these provide additional information to help characterize the Auger transitions. Auger electron angular distributions have been measured in several small molecules composed of low atomic number elements (Ref. 21 and references therein). In these studies, K-shell excitation into a state of well defined symmetry, $\Sigma$ or $\Pi$, preferentially selects molecules whose axes lie parallel or perpendicular, respectively, to the photon polarization vector. ${ }^{22}$ Thus, the photoabsorption process creates an ensemble of core excited molecules with partially aligned axes. This molecular alignment results in an anisotropic Auger electron angular distribution. $^{22}$

A different alignment mechanism, namely, the nonstatistical population of magnetic sublevels, may lead to anisotropic Auger electron angular distributions in atoms and molecules. ${ }^{23,24}$ In the present work, the effects of this latter type of alignment are explored in a molecular system, $\mathrm{CH}_{3} \mathrm{I}$. Auger transitions are usually considered within a two-step model ${ }^{25}$ where the decay of the core excited or core ionized atom is assumed to be independent of the formation process.
Within this framework, the angular distributions of Auger electrons produced by plane polarized radiation, assuming electric dipole photoabsorption by randomly oriented target atoms, may be written as ${ }^{26-29}$

$$
\frac{d W_{J_{i} \rightarrow J_{f}}(\theta)}{d \omega}=\frac{W_{J_{i} \rightarrow J_{f}}^{T}}{4 \pi}\left[1+\beta P_{2}(\cos \theta)\right],
$$

where $W_{J_{i} \rightarrow J_{f}}^{T}$ is the total Auger decay rate between initial and final states having total angular momentum $J_{i}$ and $J_{f}$, respectively, $\theta$ is the angle between the momentum of the ejected Auger electron and the polarization axis of the plane polarized radiation (the alignment axis), $\beta$ is a parameter describing the Auger electron angular distribution for that particular transition, and $P_{2}(\cos \theta)$ is the Legendre polynomial of second order. If the photoexcitation/photoionization and Auger decay are considered as two separate steps, the $\beta$ parameter can be factored into two components, ${ }^{27,28}$

$$
\beta=A_{20} \alpha_{2},
$$

where $A_{20}$ is a parameter reflecting the alignment of the core excited/ionic state produced by the photoabsorption in the first step and $\alpha_{2}$ is the intrinsic anisotropy parameter for the Auger transition.

If the total angular momentum of the core ionized state is greater than $1 / 2$, the photo-induced atomic inner-shell vacancy can be aligned. ${ }^{23,24}$ This alignment usually results in the Auger electron angular distributions being anisotropic. ${ }^{23,24}$ The alignment parameter characterizes the anisotropy of the decaying state as a result of a non-statistical population of magnetic sublevels. These populations are proportional to the photoionization partial cross sections $\sigma(J M)$, where $J$ is the total angular momentum of the intermediate state and $M$ denotes a particular magnetic sublevel. If the cross sections differ, which is usually the case, then alignment will occur, and the degree of alignment will vary with photon energy. The anisotropy parameter for a specific Auger channel depends on the total angular momentum of the initial and final states, and on the Auger decay matrix elements, and is independent of the photon energy. ${ }^{3,27}$ Auger electron angular distributions have been studied extensively for the rare gases ${ }^{3,4,23,24,29-38}$ and compared with theoretical predictions. $^{4,24,27,28,39-46}$

Berezhko et al. ${ }^{28}$ have performed a theoretical study of the photoionization induced alignment in core hole states in a range of rare gas atoms. The results from their calculations, which consider photoionization of an electron having an initial angular momentum $l$, show that the degree of alignment depends on the relative strengths of the photoionization cross sections into the two dipole allowed $l \rightarrow l-1$ and $l \rightarrow l+1$ continuum channels. In general, the $l \rightarrow l+1$ channel dominates the ionization process and the resulting alignment is rather small. However, higher values of alignment were predicted close to the ionization threshold, due to potential barrier effects, ${ }^{47}$ and in energy regions affected by a Cooper minimum, ${ }^{48}$ where the $l \rightarrow l-1$ channel dominates.

In the present work, we have recorded Auger electron spectra, using horizontally or vertically polarized radiation, 
following resonant or non-resonant excitation/ionization of the I $3 \mathrm{~d}$ level in $\mathrm{CH}_{3} \mathrm{I}$. The spectra are dominated by peaks due to the $\mathrm{M}_{45} \mathrm{~N}_{45} \mathrm{~N}_{45}$ transitions, but structure due to other Auger decay channels is also discernible. The spectra have allowed the Auger electron angular distributions to be determined, and these experimental values are compared to predictions for the corresponding transitions in Xe. Iodine lies adjacent to xenon in the periodic table, and for the valence shell, $\mathrm{CH}_{3} \mathrm{I}$ and $\mathrm{Xe}$ are isovalent.

An atomic picture description of the ionization and subsequent Auger decay relevant to our work on $\mathrm{CH}_{3} \mathrm{I}$ appears reasonable based on the binding energies of the constituent atoms. Most of the atomic orbitals of I are well isolated from those of $\mathrm{CH}_{3}$. In particular, the binding energies of the $\mathrm{C}$ and $\mathrm{H}$ orbitals are $\mathrm{C} 1 \mathrm{~s} \sim 290 \mathrm{eV}, \mathrm{C} 2 \mathrm{~s} / 2 \mathrm{p} \sim 10-20 \mathrm{eV}$, and $\mathrm{H} 1 \mathrm{~s} \sim 13 \mathrm{eV}$, while those of I are $3 \mathrm{~d}\left(\mathrm{M}_{45}\right) \sim 630 \mathrm{eV}, 4 \mathrm{~d}\left(\mathrm{~N}_{45}\right) \sim 60 \mathrm{eV}$, and $4 \mathrm{~s} / 4 \mathrm{p}\left(\mathrm{N}_{1} / \mathrm{N}_{23}\right) \sim 195 / 130 \mathrm{eV}$. Thus, the $4 \mathrm{~d}$ and more tightly bound I orbitals should not be involved significantly in the molecular bonding and should behave fairly atomically. Within the two step model of the Auger process, the first step in an Auger transition such as $\mathrm{M}_{45} \mathrm{~N}_{45} \mathrm{~N}_{45}$ involves the $\mathrm{I}_{45} \mathrm{M}_{45}$ electron-hole interaction, and this should be quite atomic-like, even in a molecule. Any molecular behavior would be due to the interaction between the escaping $\mathrm{N}_{45}$ Auger electron and the molecular charge distribution of the valence electrons and nuclei.

Additional support for our interpretation of the Auger electron angular distributions in terms of the magnetic sublevel populations is provided by our continuum multiple scattering calculations which show that, except in a small energy region close to the I $3 \mathrm{~d}$ ionization threshold, the molecular axes in the ionized state are essentially unaligned across the photon energy range considered in the present study.

Our results show that the Auger electron angular distributions, following resonant or non-resonant excitation/ionization of the I $3 \mathrm{~d}$ level in $\mathrm{CH}_{3} \mathrm{I}$, can be interpreted in a manner usually applied to atoms. The molecular environment does not appear to modify significantly either the I $3 \mathrm{~d}$ excitation/ionization dynamics ${ }^{49}$ or the magnetic sublevel populations in the intermediate state from those expected of the analogous atomic processes. The alignment responsible for the Auger electron anisotropy observed in our work therefore differs from the molecular axis alignment ${ }^{22}$ leading to the anisotropies determined in previous studies on small molecules (Ref. 21 and references therein).

\section{EXPERIMENTAL APPARATUS AND PROCEDURE}

The Auger electron spectra were recorded with a VG Scienta R4000 hemispherical electron energy analyser mounted on the soft X-ray undulator-based PLÉIADES beamline at the SOLEIL synchrotron radiation facility. Detailed descriptions of the beamline and station instrumentation have been reported previously, ${ }^{50,51}$ so only a summary is given here.

The $80 \mathrm{~mm}$ period Apple II permanent magnetic undulator was selected for the present experiment. This undulator emits radiation in the energy range $35-1000 \mathrm{eV}$ and allows the plane of polarization to be chosen to lie either parallel or perpendicular to the electron orbital plane in the storage ring. The beamline employs a modified Petersen type monochromator ${ }^{52}$ operating in the so-called fixed focus mode. The Auger electron spectra were recorded using a grating having 600 lines $/ \mathrm{mm}$ together with an exit slit width of $70 \mu \mathrm{m}$. This combination results in a theoretical optical resolution of $\sim 300 \mathrm{meV}$ at $614 \mathrm{eV}$ and $\sim 340 \mathrm{meV}$ at $666.2 \mathrm{eV}$.

The electron spectrometer was mounted in a fixed position with the electron detection axis lying perpendicular to the orbital plane in the storage ring. Photoionization occurs within a differentially pumped cell which incorporates a series of electrodes that allows compensation of the plasma potentials. ${ }^{53}$ The Auger spectra were recorded, using either horizontally or vertically polarized radiation, with an analyser pass energy of $50 \mathrm{eV}$ and a $0.5 \mathrm{~mm}$ curved entrance slit, resulting in a spectrometer resolution of $62.5 \mathrm{meV}$.

The translational Doppler broadening leads to a linewidth, $\Delta E_{\mathrm{D}}$, given by $\Delta E_{\mathrm{D}}(\mathrm{meV})=0.7215\left(E_{\mathrm{KE}} \cdot T / M\right)^{1 / 2}$, where $E_{\mathrm{KE}}$ is the kinetic energy of the electron in $\mathrm{eV}, T$ is the absolute temperature of the sample gas, and $M$ is the molecular mass expressed in atomic units. ${ }^{53}$ For an electron kinetic energy of $500 \mathrm{eV}$, which is appropriate to that of an Auger electron produced in the $\mathrm{M}_{45} \mathrm{~N}_{45} \mathrm{~N}_{45}$ decay in $\mathrm{CH}_{3} \mathrm{I}$, this corresponds to a Doppler width contribution of $\sim 23 \mathrm{meV}$.

Equation (1) is valid for $100 \%$ plane polarized radiation. In our study, the degree of polarization was determined by recording spectra encompassing the photoelectron peak due to ionization of the I $4 \mathrm{~s}$ orbital, using horizontally and vertically polarized radiation, and assuming that $\beta=2$ for this transition. The degree of polarization $(P)$ for non-completely plane polarized radiation was estimated by using the modified electron angular distribution formula ${ }^{54}$

$$
\frac{d \sigma}{d \omega}=\frac{\sigma_{\text {total }}}{4 \pi}\left[1+\frac{\beta}{4}(3 P \cos 2 \theta+1)\right],
$$

where $\sigma_{\text {total }}$ is the angle integrated cross section and the other symbols are as defined in Eq. (1). At a photon energy of $646 \mathrm{eV}$, which lies near the middle of the range relevant to the present work, we obtain $\mathrm{P}=0.991 \pm 0.005$. Hence, the assumption that completely plane polarized radiation enters the electron spectrometer appears reasonable.

The angular distribution parameters $\beta$ were obtained from the spectra recorded with the plane of polarization either parallel $\left(\theta=0^{\circ}\right)$ or perpendicular $\left(\theta=90^{\circ}\right)$ to the electron detection axis. Thus, Eq. (1) can be rewritten in the more familiar form as

$$
\beta=\frac{2\left(I_{\mathrm{par}}-I_{\mathrm{perp}}\right)}{\left(I_{\mathrm{par}}+2 I_{\mathrm{perp}}\right)},
$$

where $I_{\text {par }}$ and $I_{\text {perp }}$ are the normalized (to sample pressure, photon flux, and acquisition time) electron intensity for parallel and perpendicular polarization orientation, respectively. For the instrumentation arrangement on the PLÉIADES beamline, parallel and perpendicular orientations correspond to vertically and horizontally plane polarized radiation, respectively.

To confirm the correct operation of the beamline and spectrometer for electron angular distribution measurements, 
photoelectron spectra of the $\mathrm{Xe} 3 \mathrm{~d}$ level were measured over a photon energy range extending to $\sim 120 \mathrm{eV}$ above threshold. The $\beta$ parameters derived from these spectra were in good agreement with established values. ${ }^{55}$

The Auger electron kinetic energy scale was calibrated by recording Auger spectra due to the $\mathrm{M}_{45} \mathrm{~N}_{45} \mathrm{~N}_{45}$ transitions in xenon and using the known energies. ${ }^{56}$

The transmission efficiency of the electron analyser, at a particular kinetic energy, was determined as described by Jauhiainen et al. ${ }^{57}$ This efficiency is required to normalize the $\mathrm{M}_{45} \mathrm{~N}_{45} \mathrm{~N}_{45}$ and $\mathrm{M}_{45} \mathrm{~N}_{45} \mathrm{O}_{23}$ Auger electron spectra used to evaluate the I $3 d_{5 / 2}: I_{3} 3 d_{3 / 2}$ branching ratio (the partial photoionization cross section of the $3 \mathrm{~d}_{5 / 2}$ orbital divided by that of the $3 d_{3 / 2}$ orbital).

\section{AUGER ELECTRON PEAK FITTING PROCEDURE}

The peaks associated with the Auger electrons were fitted using the procedure described in the Appendix. This procedure allowed the intensity and the angular distribution parameter $(\beta)$ due to an individual transition to be derived through a weighted non-linear least-squares fit procedure. ${ }^{58}$ The use of a fitting routine to analyze the experimental spectra, rather than simply integrating the signal corresponding to a specific peak, allows (i) the intensities of the overlapping transitions to be separated and (ii) the final state broadening to be extracted. The Auger electron peak shape resulting from a normal transition exhibits a characteristic tailing on the high kinetic energy side, due to post-collision interaction (PCI). ${ }^{4}$ This asymmetry was incorporated into our fitting by employing the analytical line shape formula given by Armen et al. ${ }^{59}$

In our fitting of the normal Auger electron bands, initial state level widths of 461 and $473 \mathrm{meV}$ were used for the $3 \mathrm{~d}_{5 / 2}{ }^{-1}$ and $3 \mathrm{~d}_{3 / 2}{ }^{-1}$ states, respectively. These widths were obtained by averaging the widths extracted from the fits to the I 3d photoelectron peaks for photon energies between 655 and $750 \mathrm{eV}^{49}$ These widths, together with the instrumental broadening, resulted in level widths of $461 \pm 3 \mathrm{meV}$ and $455 \pm 12 \mathrm{meV}$ for the doubly ionized final states associated with the $\mathrm{M}_{45} \mathrm{~N}_{45} \mathrm{~N}_{45}$ and the $\mathrm{M}_{45} \mathrm{~N}_{45} \mathrm{O}_{23}$ Auger decays, respectively.

For resonant Auger transitions, if the photon bandwidth is narrower than the level width of the neutral excited state $\left(3 d_{5 / 2}{ }^{-1} \sigma^{*}\right.$ or $3 d_{3 / 2}{ }^{-1} \sigma^{*}$ in our case), then the initial state width can be neglected and replaced by a Gaussian representing the photon bandwidth. ${ }^{7}$ We are not aware of any reported lifetimes of the relevant core excited valence states. Our fitting procedure includes a Lorentzian for the final, singly ionized state (e.g., $4 \mathrm{~d}^{-2} \sigma^{*}$ ), as this state may decay further by additional Auger transitions. Final state broadening is usually neglected in the analysis of resonant Auger spectra involving only valence electrons due to the expected long lifetimes of the corresponding final states. Our fitting of the $3 \mathrm{~d}_{5 / 2}{ }^{-1}$ and $3 \mathrm{~d}_{3 / 2}{ }^{-1}$ spectra yields final state level widths of 480 and $469 \mathrm{meV}$, respectively. It is notable that the final state level widths extracted for the non-resonant Auger decay are similar to those extracted for the resonant Auger decay.

\section{RESULTS AND DISCUSSION}

\section{A. Overview}

An electron spectrum spanning the energy range covering all possible Auger decay channels connected with the I $3 \mathrm{~d}_{5 / 2}$ and $3 \mathrm{~d}_{3 / 2}$ core-holes, recorded at a photon energy of $666.2 \mathrm{eV}$, is plotted in Fig. 1. A spectrum covering a wider kinetic energy range has been assigned and discussed in detail by Forbes et al. ${ }^{49}$ so only those features associated with Auger transitions are considered here.

Non-resonant (normal) Auger spectra were measured at photon energies of 635.8, 640.0, 646.0, 656.0, 666.2, and $680.0 \mathrm{eV}$. Resonantly excited Auger spectra were measured at photon energies of 620.4 and $632.0 \mathrm{eV}$, coinciding with the $3 \mathrm{~d}_{5 / 2} \rightarrow \sigma^{*}$ and $3 \mathrm{~d}_{3 / 2} \rightarrow \sigma^{*}$ transitions, respectively. ${ }^{49}$ The assignments of the observed Auger electron band structure in $\mathrm{CH}_{3} \mathrm{I}$ are based on those given by Werme et al., ${ }^{56}$ Aksela et al. ${ }^{60}$ and Pulkkinen et al. ${ }^{61}$ for the corresponding bands in xenon.

\section{B. Auger electron band structure}

\section{1. $M_{45} N_{45} N_{45}$ transitions}

Auger electron spectra encompassing the $\mathrm{M}_{4} \mathrm{~N}_{45} \mathrm{~N}_{45}$ and $\mathrm{M}_{5} \mathrm{~N}_{45} \mathrm{~N}_{45}$ decay channels in $\mathrm{CH}_{3} \mathrm{I}$ are shown in Fig. 2 (a nonresonant spectrum recorded at a photon energy of $666.2 \mathrm{eV}$ and resonantly excited spectra recorded at photon energies of 620.4 and $632.0 \mathrm{eV}$, using vertically and horizontally polarized radiation). Each of the $\mathrm{M}_{4} \mathrm{~N}_{45} \mathrm{~N}_{45}$ and $\mathrm{M}_{5} \mathrm{~N}_{45} \mathrm{~N}_{45}$ Auger electron bands was fitted with 6 peaks, as shown in Fig. 3 for the normal spectrum.

The background on the high electron kinetic energy side of the Auger spectra shown in Fig. 2 is higher for that recorded with vertically polarized radiation than for that recorded with horizontally polarized radiation. This background contains a contribution from the low electron kinetic energy tail of

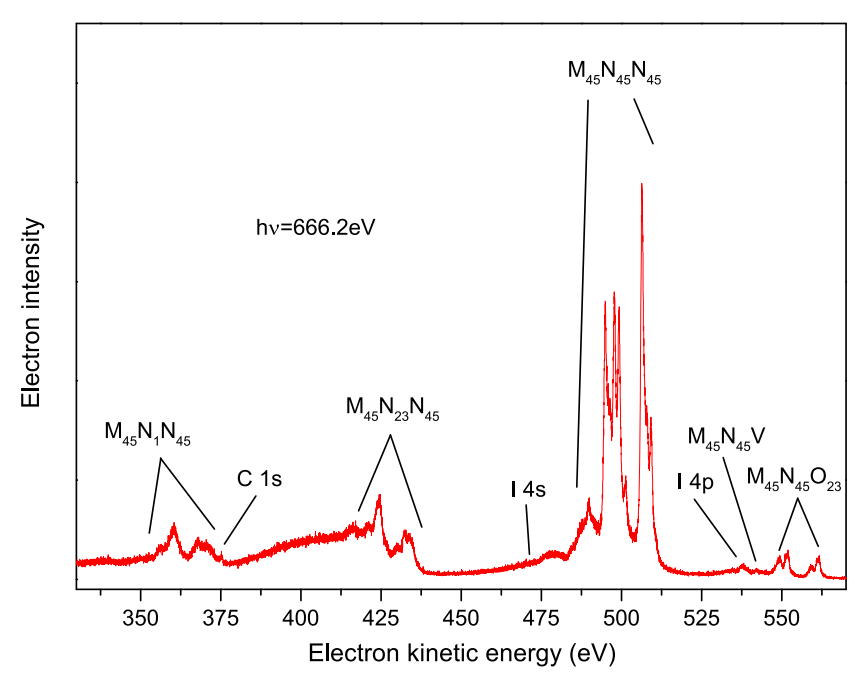

FIG. 1. The normal Auger electron spectrum of $\mathrm{CH}_{3} \mathrm{I}$ recorded at a photon energy of $666.2 \mathrm{eV}$ using horizontally polarized radiation. Most of the structure is associated with the $\mathrm{M}_{45} \mathrm{~N}_{45} \mathrm{O}_{23}, \mathrm{M}_{45} \mathrm{~N}_{45} \mathrm{~V}, \mathrm{M}_{45} \mathrm{~N}_{45} \mathrm{~N}_{45}, \mathrm{M}_{45} \mathrm{~N}_{23} \mathrm{~N}_{45}$, and $\mathrm{M}_{45} \mathrm{~N}_{1} \mathrm{~N}_{45}$ Auger transitions, although some very weak features are due to direct ionization of the I $4 \mathrm{p}, \mathrm{I} 4 \mathrm{~s}$, and $\mathrm{C} 1 \mathrm{~s}$ levels. 


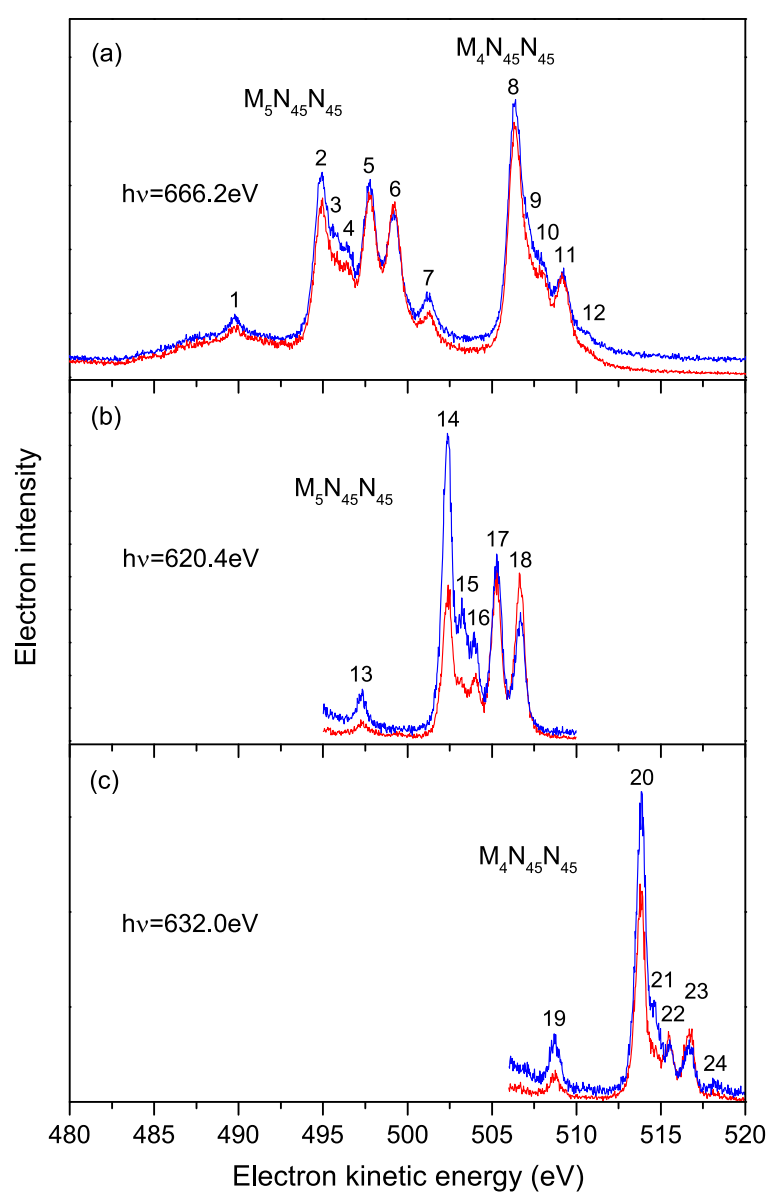

FIG. 2. Auger electron bands due to the $\mathrm{M}_{45} \mathrm{~N}_{45} \mathrm{~N}_{45}$ transitions in $\mathrm{CH}_{3} \mathrm{I}$ recorded with vertically (blue) and horizontally (red) polarized radiation. The normal spectrum (a) was measured at a photon energy of $666.2 \mathrm{eV}$. The resonantly excited spectra [(b) and (c)] were measured at photon energies of $620.4 \mathrm{eV}\left(3 \mathrm{~d}_{5 / 2} \rightarrow \sigma^{*}\right.$ transition) and $632.0 \mathrm{eV}\left(3 \mathrm{~d}_{3 / 2} \rightarrow \sigma^{*}\right.$ transition). The energies for the peaks numbered 1-24 are given in Tables I and II.

the peak due to direct ionization of the I $4 p$ orbital (having an ionization energy of $129.0 \mathrm{eV}^{49}$ ). Since the photoelectron anisotropy parameter for the I $4 p$ orbital has a value of 1.0 at $666.2 \mathrm{eV}^{49}$ the tail from the corresponding photoelectron peak provides a higher background contribution in the spectrum recorded with vertically polarized radiation than in that recorded with horizontally polarized radiation. This background contribution appears to be taken into account satisfactorily in the fitting procedure (Fig. 3) and is therefore unlikely to influence the experimentally derived $\beta$ values.

The electron kinetic energies derived from the fitting procedure are listed in Table I. The two-hole final state configuration $4 \mathrm{~d}^{-2}$ gives rise to nine terms ${ }^{60}$ but not all of these transitions were resolved in our spectra (Table I). The assignments for the peaks in $\mathrm{CH}_{3} \mathrm{I}$ have been made by analogy with the Xe Auger peaks assigned previously by Aksela et al. ${ }^{60}$ Our analysis yields electron kinetic energies of 494.72 and $506.20 \mathrm{eV}$ for peaks 2 and 8 , respectively, due to the unresolved ${ }^{1} \mathrm{D}_{2}$ and ${ }^{1} \mathrm{G}_{4}$ states. These values result in a $\mathrm{M}_{4}-\mathrm{M}_{5}$ spin-orbit splitting of $11.48 \mathrm{eV}$, which is similar to that of $11.45 \mathrm{eV}$ for the corresponding splitting in iodine vapour. $^{60}$

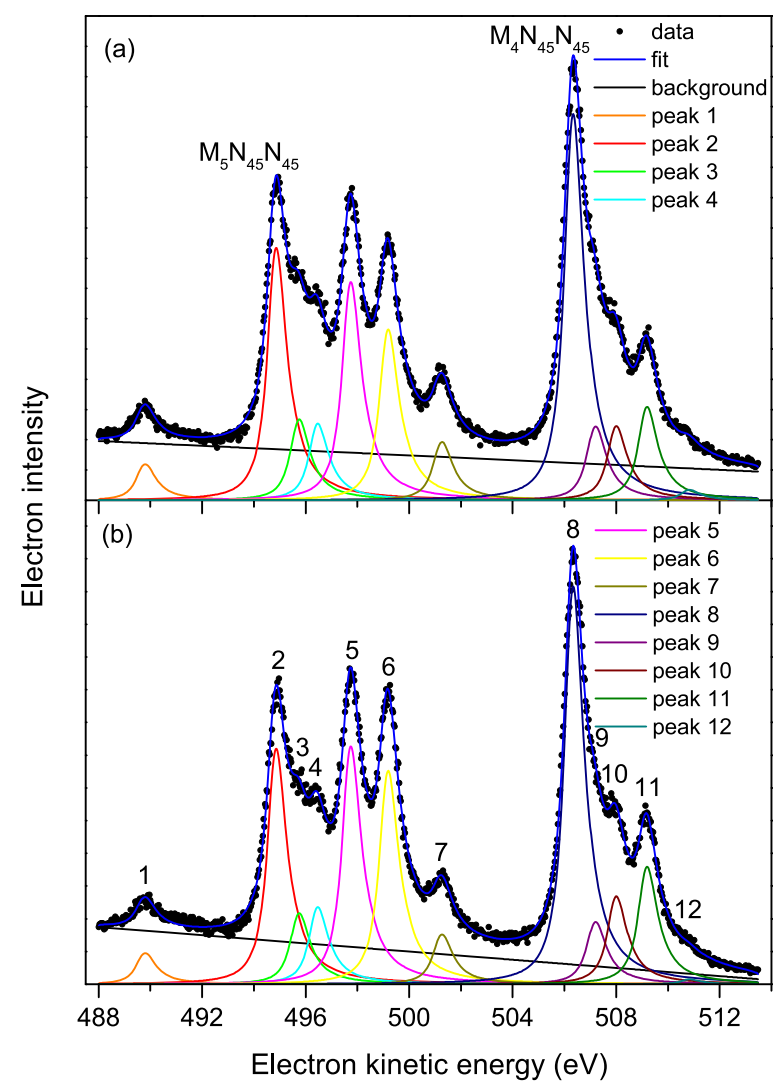

FIG. 3. Normal Auger electron bands due to the $\mathrm{M}_{45} \mathrm{~N}_{45} \mathrm{~N}_{45}$ transitions in $\mathrm{CH}_{3} \mathrm{I}$ recorded with vertically (a) and horizontally (b) polarized radiation at a photon energy of $666.2 \mathrm{eV}$. The raw and the fitted data are shown (see the text for details). The energies for the peaks numbered 1-12 are given in Table I.

Table II lists the Auger electron kinetic energies for the corresponding states following resonant excitation at 620.4 $\left(3 \mathrm{~d}_{5 / 2} \rightarrow \sigma^{*}\right)$ and $632.0 \mathrm{eV}\left(3 \mathrm{~d}_{3 / 2} \rightarrow \sigma^{*}\right)$, although in this case the final states are singly ionized with an electron promoted into the $\sigma^{*}$ valence orbital. Hence, in Table II, the states are denoted, for example, as $4 \mathrm{~d}^{-2}\left({ }^{3} \mathrm{~F}_{4}\right) \sigma^{*}$. The Auger electron kinetic energies, following resonant excitation at 620.4 or $632.0 \mathrm{eV}$, are shifted to higher energy by $\sim 7.62 \mathrm{eV}$ compared to the corresponding values in the normal Auger spectrum (Table I). See the supplementary material for fits of the resonantly excited Auger spectra.

\section{2. $\mathrm{M}_{45} \mathrm{~N}_{45} \mathrm{O}_{23}$ and $\mathrm{M}_{45} \mathrm{~N}_{45} \mathrm{~V}$ transitions}

Auger electron peaks due to the $\mathrm{M}_{45} \mathrm{~N}_{45} \mathrm{O}_{23}$ transitions appear in the kinetic energy range $\sim 545-565 \mathrm{eV}$ (Fig. 4). The structure observed in our spectra resembles to some extent that occurring in the $\mathrm{I}_{2}$ spectrum ${ }^{60}$ and that for the corresponding transitions in Xe. ${ }^{61}$ Based on the calculated $\mathrm{M}_{45} \mathrm{~N}_{45} \mathrm{O}_{123}$ spectrum in $\mathrm{Xe},{ }^{61}$ the two pairs of doublets we observe at kinetic energies of $\sim 550$ and $560 \mathrm{eV}$ in $\mathrm{CH}_{3} \mathrm{I}$ probably correspond to the $\mathrm{M}_{45} \mathrm{~N}_{45} \mathrm{O}_{23}$ transitions. In our fitting, five peaks are allocated to each of the $\mathrm{M}_{4} \mathrm{~N}_{45} \mathrm{O}_{23}$ and $\mathrm{M}_{5} \mathrm{~N}_{45} \mathrm{O}_{23}$ transitions, following the assignments of the analogous structure in xenon. ${ }^{60,61}$ The electron kinetic energies derived from this fitting to the normal Auger spectrum recorded at a photon energy of $666.2 \mathrm{eV}$ are listed in Table III. In the resonantly excited spectrum recorded at a photon energy of $620.4 \mathrm{eV}$ 
TABLE I. Experimental Auger electron kinetic energies and intrinsic anisotropy parameters for the iodine $\mathrm{M}_{45} \mathrm{~N}_{45} \mathrm{~N}_{45}$ transitions in $\mathrm{CH}_{3} \mathrm{I}$, and calculated intrinsic anisotropy parameters for the $\mathrm{M}_{45} \mathrm{~N}_{45} \mathrm{~N}_{45}$ transitions in $\mathrm{Xe}$.

\begin{tabular}{|c|c|c|c|c|c|c|}
\hline Transition & $\begin{array}{l}\text { Final } \\
\text { state }\end{array}$ & $\begin{array}{c}\text { Peak } \\
\text { number }\end{array}$ & $\begin{array}{c}\text { Auger electron kinetic } \\
\text { energy }(\mathrm{eV})^{\mathrm{a}}\end{array}$ & $\begin{array}{c}\beta \\
(\text { expt. })^{\mathrm{b}, \mathrm{c}}\end{array}$ & $\begin{array}{c}\alpha_{2} \\
(\operatorname{expt} .)^{\mathrm{c}, \mathrm{d}}\end{array}$ & $\begin{array}{c}\alpha_{2} \\
(\mathrm{calc})^{\mathrm{e}}\end{array}$ \\
\hline \multirow[t]{6}{*}{$\mathrm{M}_{5} \mathrm{~N}_{45} \mathrm{~N}_{45}$} & ${ }^{1} \mathrm{~S}_{0}$ & 1 & 489.65 & $0.151 \pm 0.022$ & $-0.718 \pm 0.103$ & -1.069 \\
\hline & $\begin{array}{l}{ }^{1} \mathrm{D}_{2} \\
{ }^{1} \mathrm{G}_{4}\end{array}$ & 2 & 494.72 & $0.059 \pm 0.004$ & $-0.279 \pm 0.018$ & $\begin{array}{l}-0.131 \\
-0.719\end{array}$ \\
\hline & $\begin{array}{l}{ }^{3} \mathrm{P}_{1} \\
{ }^{3} \mathrm{P}_{0}\end{array}$ & 3 & 495.60 & $0.173 \pm 0.012$ & $-0.822 \pm 0.058$ & $\begin{array}{l}-0.748 \\
-1.069\end{array}$ \\
\hline & ${ }^{3} \mathrm{P}_{2}$ & 4 & 496.33 & $0.076 \pm 0.011$ & $-0.364 \pm 0.052$ & -0.389 \\
\hline & $\begin{array}{l}{ }^{3} \mathrm{~F}_{3} \\
{ }^{3} \mathrm{~F}_{2}\end{array}$ & 5 & 497.60 & $0.013 \pm 0.004$ & $-0.061 \pm 0.017$ & $\begin{array}{r}0.331 \\
-0.222\end{array}$ \\
\hline & ${ }^{3} \mathrm{~F}_{4}$ & 6 & 499.04 & $-0.072 \pm 0.004$ & $0.345 \pm 0.019$ & 0.378 \\
\hline \multirow[t]{6}{*}{$\mathrm{M}_{4} \mathrm{~N}_{45} \mathrm{~N}_{45}$} & ${ }^{1} \mathrm{~S}_{0}$ & 7 & 501.13 & $0.172 \pm 0.011$ & $-0.862 \pm 0.053$ & -1.000 \\
\hline & $\begin{array}{l}{ }^{1} \mathrm{D}_{2} \\
{ }^{1} \mathrm{G}_{4}\end{array}$ & 8 & 506.20 & $0.038 \pm 0.003$ & $-0.191 \pm 0.014$ & $\begin{array}{r}0.050 \\
-0.653\end{array}$ \\
\hline & $\begin{array}{l}{ }^{3} \mathrm{P}_{1} \\
{ }^{3} \mathrm{P}_{0}\end{array}$ & 9 & 507.06 & $0.150 \pm 0.018$ & $-0.750 \pm 0.089$ & $\begin{array}{l}-0.799 \\
-1.000\end{array}$ \\
\hline & ${ }^{3} \mathrm{P}_{2}$ & 10 & 507.87 & $-0.079 \pm 0.011$ & $0.393 \pm 0.053$ & 0.378 \\
\hline & $\begin{array}{l}{ }^{3} \mathrm{~F}_{3} \\
{ }^{3} \mathrm{~F}_{2}\end{array}$ & 11 & 509.06 & $-0.104 \pm 0.006$ & $0.521 \pm 0.031$ & $\begin{array}{l}0.432 \\
0.738\end{array}$ \\
\hline & ${ }^{3} \mathrm{~F}_{4}$ & 12 & 510.70 & $0.500 \pm 0.077$ & $-2.502 \pm 0.385$ & -0.826 \\
\hline
\end{tabular}

${ }^{a}$ Auger electron kinetic energies obtained by fitting the spectrum recorded at a photon energy of $666.2 \mathrm{eV}$.

${ }^{\mathrm{b}}$ The average $\beta$ parameter for the $\mathrm{M}_{5}\left(3 \mathrm{~d}_{5 / 2}{ }^{-1}\right)$ core hole was obtained by averaging the values obtained at six photon energies $(635.8,640.0,646.0,656.0,666.2$, and $680.0 \mathrm{eV})$. The average $\beta$ parameter for the $\mathrm{M}_{4}\left(3 \mathrm{~d}_{3 / 2}{ }^{-1}\right)$ core hole was obtained by averaging the values obtained at three photon energies $(656.0,666.2$, and $680.0 \mathrm{eV})$.

${ }^{\mathrm{c}}$ The quoted uncertainty is due only to electron counting statistics and peak fitting, and does not take any systematic errors into account.

${ }^{\mathrm{d}}$ The experimental $\alpha_{2}$ parameters were obtained from the experimental $\beta$ parameters using theoretical values $A_{20}\left(\mathrm{D}_{5 / 2}\right)=-0.21$ and $A_{20}\left(\mathrm{D}_{3 / 2}\right)=-0.20$.

${ }^{\mathrm{e}}$ Calculated $\alpha_{2}$ parameters for the $\mathrm{M}_{45} \mathrm{~N}_{45} \mathrm{~N}_{45}$ transitions in xenon. ${ }^{42}$

(not shown), a peak appearing at an electron kinetic energy of $558.35 \mathrm{eV}$ probably corresponds to the unresolved contributions from peaks 28 and 29 (Table III). Thus, the $\mathrm{M}_{45} \mathrm{~N}_{45} \mathrm{O}_{23}$ peaks are shifted by $\sim 6.9 \mathrm{eV}$ to higher energy in the resonantly excited Auger spectrum.

The broader and weaker structure occurring between $\sim 530$ and $545 \mathrm{eV}$ (Fig. 4) probably arises from transitions $\left(\mathrm{M}_{45} \mathrm{~N}_{45} \mathrm{~V}\right)$ involving the molecular valence orbitals. Note that the relatively intense peak at $\sim 537.8 \mathrm{eV}$ in the spectrum recorded at a photon energy of $666.2 \mathrm{eV}$ contains a contribution from photoelectrons emitted from the I $4 \mathrm{p}$ level. ${ }^{49}$ A section of the electron spectrum, which should not be affected by direct ionization, recorded at a photon energy of $646.0 \mathrm{eV}$, is also shown in Fig. 4. Three broad peaks are observed at kinetic energies of 534.5, 538.7, and $542.1 \mathrm{eV}$ which we associate with Auger decays involving the valence orbitals.

In order to obtain a better understanding of the Auger decays in $\mathrm{CH}_{3} \mathrm{I}$ involving the iodine $\mathrm{O}$-shell, the atomic populations of the valence orbitals were determined by MP2 calculations using Gaussian 09 with a 3-21G basis set. ${ }^{62}$ The results indicate that the two components of the 2e (HOMO [highest occupied molecular orbital]) valence orbital are essentially nearly pure iodine $\mathrm{p}_{\mathrm{x}}$ and $\mathrm{p}_{\mathrm{y}}$ atomic orbitals. This is consistent with photoelectron studies which show that the observed $(628 \mathrm{meV})$ spin-orbit splitting in the $(2 \mathrm{e})^{-1}$ band is similar to that $(629 \mathrm{meV})$ for atomic iodine. ${ }^{63}$ Furthermore, the photoelectron angular distribution associated with the $2 \mathrm{e}$ orbital exhibits an energy dependence reminiscent of that predicted for the atomic I 5p Cooper minimum. ${ }^{64}$

Our calculations also show that there is no I 5 s atomic-like molecular orbital in $\mathrm{CH}_{3} \mathrm{I}$. The I 5 s orbital can interact with the $\mathrm{C}$, and possibly the $\mathrm{H}$, atomic orbitals in all the molecular orbitals of $a_{1}$ symmetry. The $1 a_{1}$ and $2 a_{1}$ valence orbitals may be regarded as a $\sigma / \sigma^{*}$ pair formed by the interacting I $5 \mathrm{~s}$ and $\mathrm{C} 2 \mathrm{~s} / 2 \mathrm{p}_{\mathrm{z}}$ atomic orbitals, and the $3 \mathrm{a}_{1}$ (HOMO-1) orbital may be characterized as a $\mathrm{C}-\mathrm{I} \sigma$-bonding orbital. These characterizations of the valence shell molecular orbitals in terms of the atomic contributions are in accord with those reported by Pernpointner et al. ${ }^{65}$ Plots of the valence orbitals are available in the supplementary material. These predictions rationalize the 
TABLE II. Experimental Auger electron kinetic energies and intrinsic anisotropy parameters for the iodine $\mathrm{M}_{45} \mathrm{~N}_{45} \mathrm{~N}_{45}$ transitions in $\mathrm{CH}_{3} \mathrm{I}$ following resonant excitation at $620.4 \mathrm{eV}\left(3 \mathrm{~d}_{5 / 2} \rightarrow \sigma^{*}\right)$ or $632.0 \mathrm{eV}$ $\left(3 d_{3 / 2} \rightarrow \sigma^{*}\right)$.

\begin{tabular}{|c|c|c|c|c|}
\hline $\begin{array}{l}\text { Transition and } \\
\text { final state }\end{array}$ & $\begin{array}{c}\text { Peak } \\
\text { number }\end{array}$ & $\begin{array}{c}\text { Auger electron kinetic } \\
\text { energy }(\mathrm{eV})\end{array}$ & $\begin{array}{c}\beta \\
(\text { expt. })^{\mathrm{a}}\end{array}$ & $\begin{array}{c}\alpha_{2} \\
{\left(\text { expt. }^{\mathrm{a}, \mathrm{b}}\right.}\end{array}$ \\
\hline \multicolumn{5}{|c|}{ I $3 d_{5 / 2}^{-1} \sigma^{*} \rightarrow 4 d^{-2} \sigma^{* c}$} \\
\hline $4 \mathrm{~d}^{-2}\left({ }^{1} \mathrm{~S}_{0}\right) \sigma^{*}$ & 13 & 497.26 & $0.741 \pm 0.056$ & $-0.524 \pm 0.040$ \\
\hline $4 \mathrm{~d}^{-2}\left({ }^{1} \mathrm{D}_{2}+{ }^{1} \mathrm{G}_{4}\right) \sigma^{*}$ & 14 & 502.36 & $0.520 \pm 0.016$ & $-0.368 \pm 0.011$ \\
\hline $4 \mathrm{~d}^{-2}\left({ }^{3} \mathrm{P}_{0,1}\right) \sigma^{*}$ & 15 & 503.26 & $0.624 \pm 0.044$ & $-0.441 \pm 0.031$ \\
\hline $4 \mathrm{~d}^{-2}\left({ }^{3} \mathrm{P}_{2}\right) \sigma^{*}$ & 16 & 503.98 & $0.241 \pm 0.037$ & $-0.170 \pm 0.026$ \\
\hline $4 \mathrm{~d}^{-2}\left({ }^{3} \mathrm{~F}_{2}+{ }^{3} \mathrm{~F}_{3}\right) \sigma^{*}$ & 17 & 505.28 & $0.042 \pm 0.015$ & $-0.030 \pm 0.011$ \\
\hline $4 d^{-2}\left({ }^{3} F_{4}\right) \sigma^{*}$ & 18 & 506.66 & $-0.189 \pm 0.015$ & $0.134 \pm 0.011$ \\
\hline \multicolumn{5}{|c|}{ I $3 d_{3 / 2} \sigma^{*} \rightarrow 4 d^{-2} \sigma^{* d}$} \\
\hline $4 \mathrm{~d}^{-2}\left({ }^{1} \mathrm{~S}_{0}\right) \sigma^{*}$ & 19 & 508.70 & $0.595 \pm 0.042$ & $-0.421 \pm 0.029$ \\
\hline $4 \mathrm{~d}^{-2}\left({ }^{1} \mathrm{D}_{2}+{ }^{1} \mathrm{G}_{4}\right) \sigma^{*}$ & 20 & 513.82 & $0.260 \pm 0.014$ & $-0.184 \pm 0.010$ \\
\hline $4 \mathrm{~d}^{-2}\left({ }^{3} \mathrm{P}_{0,1}\right) \sigma^{*}$ & 21 & 514.69 & $0.507 \pm 0.061$ & $-0.359 \pm 0.043$ \\
\hline $4 \mathrm{~d}^{-2}\left({ }^{3} \mathrm{P}_{2}\right) \sigma^{*}$ & 22 & 515.51 & $-0.253 \pm 0.036$ & $0.179 \pm 0.025$ \\
\hline $4 \mathrm{~d}^{-2}\left({ }^{3} \mathrm{~F}_{2}+{ }^{3} \mathrm{~F}_{3}\right) \sigma^{*}$ & 23 & 516.68 & $-0.234 \pm 0.026$ & $0.165 \pm 0.018$ \\
\hline $4 d^{-2}\left({ }^{3} F_{4}\right) \sigma^{*}$ & 24 & 518.29 & $0.534 \pm 0.159$ & $-0.378 \pm 0.112$ \\
\hline
\end{tabular}

${ }^{\mathrm{a}}$ The quoted uncertainty is due only to electron counting statistics and peak fitting, and does not take any systematic errors into account.

${ }^{\mathrm{b}}$ The experimental $\alpha_{2}$ parameters were obtained from the experimental $\beta$ parameters using $A_{20}=-\sqrt{2}$.

${ }^{\mathrm{c}}$ Auger electron kinetic energies and $\beta$ parameters obtained by fitting the resonantly excited spectrum recorded at a photon energy of $620.4 \mathrm{eV}$.

${ }^{\mathrm{d}}$ Auger electron kinetic energies and $\beta$ parameters obtained by fitting the resonantly excited spectrum recorded at a photon energy of $632.0 \mathrm{eV}$.

evident similarity between the $\mathrm{M}_{45} \mathrm{~N}_{45} \mathrm{O}_{23}$ transitions in $\mathrm{CH}_{3} \mathrm{I}$ and the corresponding transitions in $\mathrm{Xe}$, and also indicate that the structure observed between $\sim 530$ and $545 \mathrm{eV}$ should be attributed to the $\mathrm{M}_{45} \mathrm{~N}_{45} \mathrm{~V}$ transitions.

\section{3. $M_{45} N_{23} N_{45}$ transitions}

Figure 5 shows the non-resonant $(666.2 \mathrm{eV})$ and resonant $(620.4 \mathrm{eV})$ Auger spectra in the electron kinetic energy

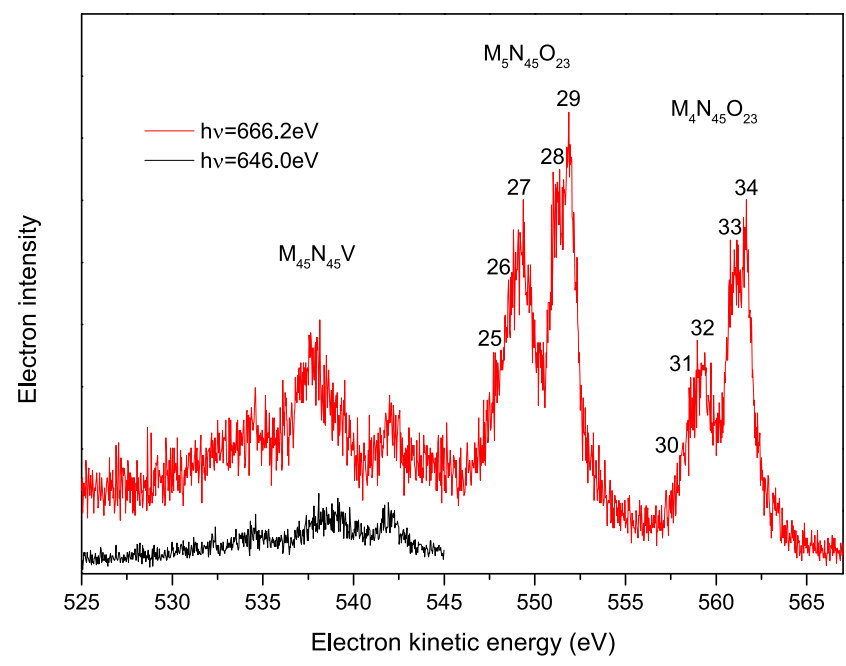

FIG. 4. The normal Auger spectrum of $\mathrm{CH}_{3} \mathrm{I}$ showing bands due to the $\mathrm{M}_{45} \mathrm{~N}_{45} \mathrm{O}_{23}$ and $\mathrm{M}_{45} \mathrm{~N}_{45} \mathrm{~V}$ transitions. The spectrum (red) was recorded at a photon energy of $666.2 \mathrm{eV}$ using horizontally polarized radiation. The energies for the peaks numbered 25-34 are given in Table III. A section of the spectrum (black) recorded at a photon energy of $646.0 \mathrm{eV}$ using horizontally polarized radiation is also shown.
TABLE III. Auger electron kinetic energies for the iodine $\mathrm{M}_{45} \mathrm{~N}_{45} \mathrm{O}_{23}$, $\mathrm{M}_{45} \mathrm{~N}_{23} \mathrm{~N}_{45}$, and $\mathrm{M}_{45} \mathrm{~N}_{1} \mathrm{~N}_{45}$ transitions in $\mathrm{CH}_{3} \mathrm{I}$.

\begin{tabular}{|c|c|c|}
\hline Transition & $\begin{array}{c}\text { Peak } \\
\text { number }\end{array}$ & $\begin{array}{c}\text { Auger electron kinetic } \\
\text { energy }(\mathrm{eV})\end{array}$ \\
\hline \multirow[t]{5}{*}{$\mathrm{M}_{5} \mathrm{~N}_{45} \mathrm{O}_{23}$} & 25 & 547.82 \\
\hline & 26 & 548.74 \\
\hline & 27 & 549.46 \\
\hline & 28 & 551.10 \\
\hline & 29 & 551.87 \\
\hline \multirow[t]{5}{*}{$\mathrm{M}_{4} \mathrm{~N}_{45} \mathrm{O}_{23}$} & 30 & 558.02 \\
\hline & 31 & 558.69 \\
\hline & 32 & 559.29 \\
\hline & 33 & 560.79 \\
\hline & 34 & 561.55 \\
\hline \multirow[t]{5}{*}{$\mathrm{M}_{5} \mathrm{~N}_{23} \mathrm{~N}_{45}$} & 35 & 415.6 \\
\hline & 36 & 417.0 \\
\hline & 37 & 421.1 \\
\hline & 38 & 423.72 \\
\hline & 39 & 424.47 \\
\hline \multirow[t]{4}{*}{$\mathrm{M}_{4} \mathrm{~N}_{23} \mathrm{~N}_{45}$} & 40 & 430.3 \\
\hline & 41 & 432.36 \\
\hline & 42 & 433.80 \\
\hline & 43 & 434.73 \\
\hline \multirow[t]{2}{*}{$\mathrm{M}_{5} \mathrm{~N}_{1} \mathrm{~N}_{45}$} & 44 & 356.4 \\
\hline & 45 & 360.4 \\
\hline \multirow[t]{2}{*}{$\mathrm{M}_{4} \mathrm{~N}_{1} \mathrm{~N}_{45}$} & 46 & 367.9 \\
\hline & 47 & 370.5 \\
\hline
\end{tabular}




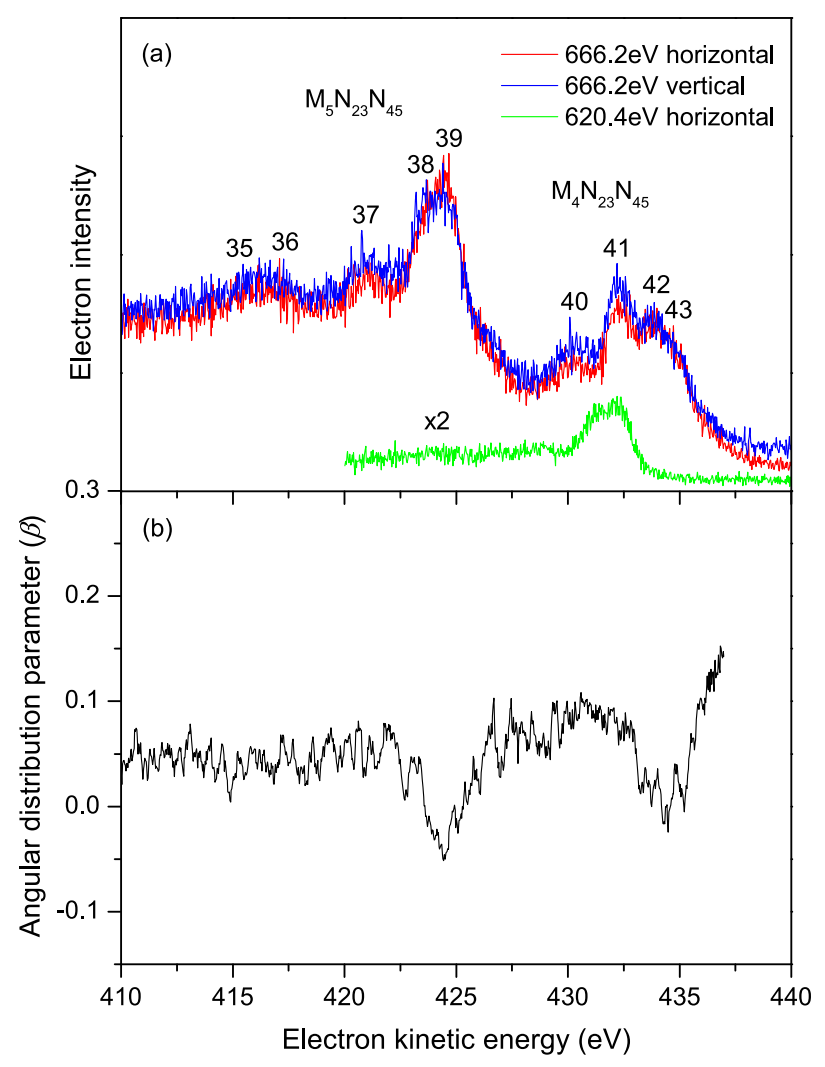

FIG. 5. (a) Auger electron bands due to the $\mathrm{M}_{45} \mathrm{~N}_{23} \mathrm{~N}_{45}$ transitions in $\mathrm{CH}_{3} \mathrm{I}$ recorded at $666.2 \mathrm{eV}$ using vertically (blue) and horizontally (red) polarized radiation and at $620.4 \mathrm{eV}$ using horizontally (green) polarized radiation. The energies for the peaks numbered 35-43 are given in Table III. (b) The angular distribution parameter for the $\mathrm{M}_{45} \mathrm{~N}_{23} \mathrm{~N}_{45}$ transitions derived from the vertically and horizontally polarized spectra measured at $666.2 \mathrm{eV}$. A 10-point moving average has been applied to improve the signal-to-noise ratio. $\beta$ values are omitted in regions of low electron intensity.

range $410-440 \mathrm{eV}$. The observed structure is associated with the $\mathrm{M}_{45} \mathrm{~N}_{23} \mathrm{~N}_{45}$ transitions. The resonantly excited spectrum recorded with vertically polarized radiation (not shown) is dominated by a peak due to photoionization of the I $4 \mathrm{~s}$ level. However, this peak is absent in the spectrum recorded with horizontally polarized radiation because the $\beta$ parameter for the $4 \mathrm{~s}$ electron is $\sim 2$. Aksela et al. ${ }^{60}$ and Tulkki et al. ${ }^{44}$ have calculated the Auger electron energies and relative line intensities for the $\mathrm{M}_{45} \mathrm{~N}_{23} \mathrm{~N}_{45}$ transitions in xenon. However, correlating these theoretical results with the bands observed in $\mathrm{CH}_{3} \mathrm{I}$ is difficult due, at least in part, to inconsistencies in the predicted ordering of the final states. ${ }^{44,60}$

Forbes et al. ${ }^{49}$ have shown that the photoelectron band associated with the I $4 \mathrm{p}$ orbital in $\mathrm{CH}_{3} \mathrm{I}$ has an unusual appearance. Instead of a well resolved spin-orbit doublet, due to the $4 \mathrm{p}^{5}{ }^{2} \mathrm{P}_{3 / 2}$ and ${ }^{2} \mathrm{P}_{1 / 2}$ ionization limits, the spectrum exhibits a single structured peak together with an extended continuum. This anomalous intensity distribution was ascribed to the rapid decay of the inner-shell hole state through Coster-Kronig and super-Coster-Kronig processes, as previously observed ${ }^{66}$ and predicted ${ }^{67}$ for the $4 p$ orbital in xenon. It is likely that the numerous excited states responsible for the continuum associated with the $\mathrm{I} 4 \mathrm{p}$ photoelectron band in $\mathrm{CH}_{3} \mathrm{I}$ also account for the continuum underlying the Auger electron peaks due to the $\mathrm{M}_{45} \mathrm{~N}_{23} \mathrm{~N}_{45}$ transitions.
The normal Auger spectrum recorded at a photon energy of $666.2 \mathrm{eV}$ appears to show five peaks due to the $\mathrm{M}_{5} \mathrm{~N}_{23} \mathrm{~N}_{45}$ transitions and four peaks due to the $\mathrm{M}_{4} \mathrm{~N}_{23} \mathrm{~N}_{45}$ transitions (Table III). In the resonantly excited spectrum recorded at $620.4 \mathrm{eV}$, two peaks are observed at 431.39 and $432.11 \mathrm{eV}$. These correspond to peaks 38 and 39, associated with the $\mathrm{M}_{5} \mathrm{~N}_{23} \mathrm{~N}_{45}$ transitions, shifted to higher kinetic energies by 7.67 and $7.64 \mathrm{eV}$, respectively.

\section{4. $M_{45} N_{1} N_{45}$ transitions}

The electron kinetic energy range $\sim 350-380 \mathrm{eV}$ encompasses Auger electron bands due to the $\mathrm{M}_{45} \mathrm{~N}_{1} \mathrm{~N}_{45}$ transitions. Figure 6(a) shows the normal spectrum $(666.2 \mathrm{eV})$ and the resonantly excited spectra $(620.4$ and $632.0 \mathrm{eV})$ recorded with horizontally polarized radiation. In the corresponding spectra recorded with vertically polarized radiation, structure is observed due to photoionization of the $\mathrm{C} 1 \mathrm{~s}$ level and the associated satellite states. However, the $\beta$ parameters for the $\mathrm{C} 1 \mathrm{~s}$ main-line and the shake-up spectrum are close to 2 , whereas the $\beta$ parameters associated with the $\mathrm{M}_{45} \mathrm{~N}_{1} \mathrm{~N}_{45}$ Auger electrons are approximately zero. Hence, a comparison between the spectra recorded with vertically or horizontally polarized radiation allows the Auger electron peaks to be
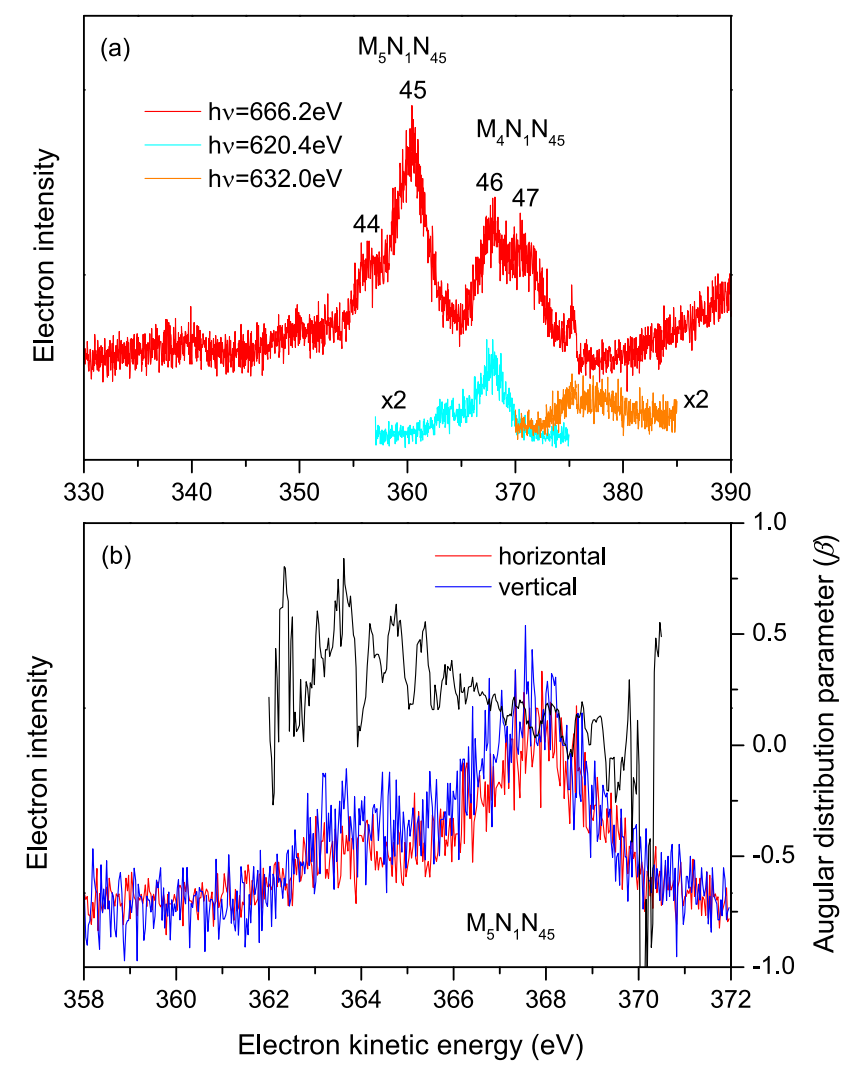

FIG. 6. (a) Auger electron bands due to the $\mathrm{M}_{45} \mathrm{~N}_{1} \mathrm{~N}_{45}$ transitions in $\mathrm{CH}_{3} \mathrm{I}$ recorded with horizontally polarized radiation. The normal spectrum [red] was measured at a photon energy of $666.2 \mathrm{eV}$. The resonantly excited spectra were measured at photon energies of $620.4 \mathrm{eV}$ [cyan] ( $3 \mathrm{~d}_{5 / 2} \rightarrow \sigma^{*}$ transition) and $632.0 \mathrm{eV}$ [orange] $\left(3 \mathrm{~d}_{3 / 2} \rightarrow \sigma^{*}\right.$ transition). The energies for the peaks numbered $44-47$ are given in Table III. (b) The angular distribution parameter [black] (smoothed) for the $\mathrm{M}_{5} \mathrm{~N}_{1} \mathrm{~N}_{45}$ transition derived from the vertically [blue] and horizontally [red] polarized spectra measured at $620.4 \mathrm{eV}$. A $10-$ point moving average was applied to improve the signal-to-noise ratio. $\beta$ values are omitted in regions of low electron intensity. 
distinguished. In the normal Auger electron spectrum, we associate two broad peaks with each of the $\mathrm{M}_{4} \mathrm{~N}_{1} \mathrm{~N}_{45}$ and $\mathrm{M}_{5} \mathrm{~N}_{1} \mathrm{~N}_{45}$ transitions. The electron kinetic energies corresponding to these four peaks are given in Table III. In the resonantly excited spectrum recorded at a photon energy of $620.4 \mathrm{eV}$, the peaks observed at 363.7 and $367.8 \mathrm{eV}$ correspond to peaks 44 and 45 shifted by 7.3 and $7.4 \mathrm{eV}$, respectively, to higher kinetic energy. Similarly, in the resonant spectrum recorded at $632.0 \mathrm{eV}$, peaks 46 and 47 are shifted by 7.4 and $7.2 \mathrm{eV}$, respectively, and appear at kinetic energies of $375.3 \mathrm{eV}$ and $377.7 \mathrm{eV}$.

In regard to the possible assignment of the doublet associated with the $\mathrm{M}_{5} \mathrm{~N}_{1} \mathrm{~N}_{45}$ transitions, it is conceivable that the more intense component occurring at the higher kinetic energy $(360.4 \mathrm{eV})$ should be associated with the ${ }^{3} \mathrm{D}_{3}$ and ${ }^{3} \mathrm{D}_{2}$ states which, in xenon, ${ }^{44}$ are calculated to have high relative intensities, whilst the weaker component, observed at $356.4 \mathrm{eV}$, should be associated with the ${ }^{3} \mathrm{D}_{1}$ and ${ }^{1} \mathrm{D}_{2}$ states which are predicted to have lower relative intensities. ${ }^{44}$

\section{5. $M_{45}$ Auger electron and I $3 d_{5 / 2,3 / 2}$ photoelectron branching ratios}

The Auger electron spectra were used to obtain the $\mathrm{M}_{4} \mathrm{~N}_{45} \mathrm{~N}_{45}: \mathrm{M}_{5} \mathrm{~N}_{45} \mathrm{~N}_{45}$ and the $\mathrm{M}_{4} \mathrm{~N}_{45} \mathrm{O}_{23}: \mathrm{M}_{5} \mathrm{~N}_{45} \mathrm{O}_{23}$ branching ratios. These ratios are plotted in Fig. 7, together with that for the $\mathrm{I}_{3 \mathrm{~d}_{5 / 2}}: 3 \mathrm{~d}_{3 / 2}$ photoelectrons. ${ }^{49}$ The three branching ratios exhibit a similar variation as a function of energy, as expected.

\section{Shift in Auger electron kinetic energies between normal and resonantly excited spectra}

Our experimental results show that the Auger electron kinetic energies associated with the $\mathrm{M}_{45} \mathrm{~N}_{45} \mathrm{~N}_{45}, \mathrm{M}_{45} \mathrm{~N}_{23} \mathrm{~N}_{45}$, and $\mathrm{M}_{45} \mathrm{~N}_{1} \mathrm{~N}_{45}$ transitions are shifted to higher energy by $\sim 7.6,7.7$, and $7.3 \mathrm{eV}$ in the resonantly excited spectra compared to the corresponding energies in the normal spectra. A shift is also observed, albeit a smaller one $(6.9 \mathrm{eV})$, for the $\mathrm{M}_{45} \mathrm{~N}_{45} \mathrm{O}_{23}$ transitions. These shifts arise from the screening

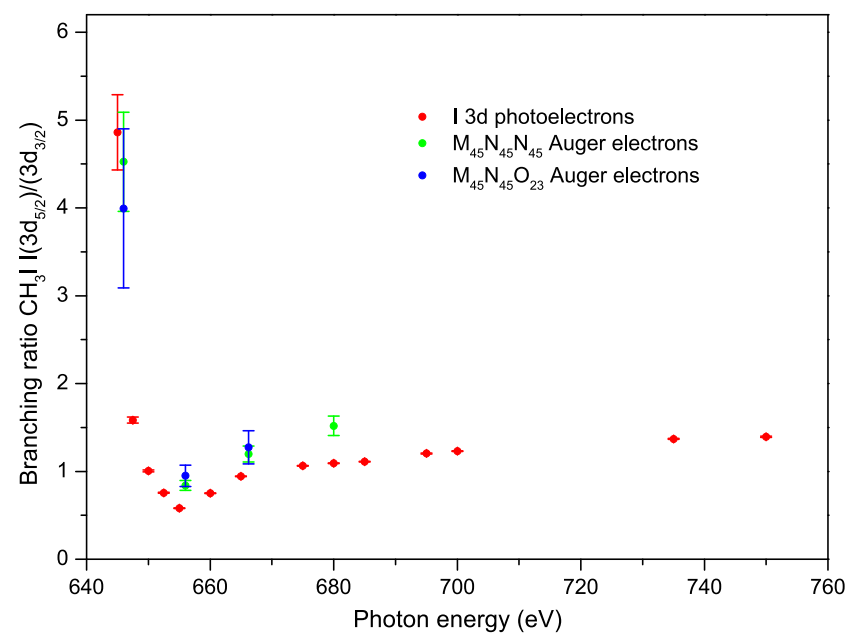

FIG. 7. Branching ratios for the $\mathrm{M}_{4} \mathrm{~N}_{45} \mathrm{~N}_{45}: \mathrm{M}_{5} \mathrm{~N}_{45} \mathrm{~N}_{45}$ (green) and the $\mathrm{M}_{4} \mathrm{~N}_{45} \mathrm{O}_{23}: \mathrm{M}_{5} \mathrm{~N}_{45} \mathrm{O}_{23}$ (blue) Auger transitions and the I $3 \mathrm{~d}_{5 / 2}: 3 \mathrm{~d}_{3 / 2}$ (red) photoelectrons.
TABLE IV. Orbital ionization energies for I 3d ionized and I 3d excited states in $\mathrm{CH}_{3} \mathrm{I}$.

\begin{tabular}{lcc}
\hline \hline & \multicolumn{2}{c}{ Ionization energy $(\mathrm{eV})$} \\
\cline { 2 - 3 } Orbital & I 3d ionized state & $\mathrm{a}$ \\
\hline $\mathrm{N}_{1}$ & 199.31 & 191.65 \\
$\mathrm{~N}_{23}$ & 154.41 & 146.85 \\
$\mathrm{~N}_{45}$ & 78.10 & 70.46 \\
$1 \mathrm{a}_{1}$ & 28.05 & 20.37 \\
$2 \mathrm{a}_{1}$ & 31.20 & 23.50 \\
$1 \mathrm{e}$ & 21.62 & 14.17 \\
$3 \mathrm{a}_{1}$ & 20.80 & 12.99 \\
$2 \mathrm{e}$ & 18.71 & 11.68 \\
\hline \hline
\end{tabular}

${ }^{\mathrm{a}}$ The ionized state corresponds to a hole in the I $3 \mathrm{~d}$ shell.

${ }^{\mathrm{b}}$ The neutral excited state corresponds to an I $3 \mathrm{~d}$ electron excited into the $\sigma^{*}$ orbital.

provided by the electron excited into the $\sigma^{*}$ orbital. We have calculated the orbital ionization energies of the methyl iodide ion with a hole in the I $3 \mathrm{~d}$ shell and also those of neutral methyl iodide having a $3 \mathrm{~d}$ electron excited into the $\sigma^{*}$ orbital. The transitions energies were determined using a $\Delta$ self-consistent field approach with the ground and excited state energies computed using density functional theory with the hybrid 3-parameter Becke exchange and Lee, Yang and Parr correlation functional $(\mathrm{B} 3 \mathrm{LYP})^{68}$ with the $6-311 \mathrm{G}^{* *}$ basis set. The maximum overlap method ${ }^{69,70}$ was used to prevent variational collapse in the calculation of the core excited states. All calculations were performed with the Q-Chem software package. ${ }^{71}$ A summary of the relevant energies is given in Table IV, with full details of additional energies and orbital plots being available in the supplementary material.

Using our experimental value of $626.8 \mathrm{eV}$ for the ionization energy of the $3 \mathrm{~d}_{5 / 2}$ orbital, ${ }^{49}$ and the calculated ionization energy of $78.1 \mathrm{eV}$ for the $\mathrm{N}_{45}$ orbital in ionized $\mathrm{CH}_{3} \mathrm{I}$ with a $3 \mathrm{~d}$ hole, results in an estimated Auger electron kinetic energy of $470.6 \mathrm{eV}$ for the $\mathrm{M}_{5} \mathrm{~N}_{45} \mathrm{~N}_{45}$ transitions. Similarly, using our experimental value of $620.4 \mathrm{eV}$ for the excitation energy of the $3 \mathrm{~d}_{5 / 2} \rightarrow \sigma^{*}$ transition, and the calculated ionization energy of $70.5 \mathrm{eV}$ for the $\mathrm{N}_{45}$ orbital in $\mathrm{CH}_{3} \mathrm{I}$ with a $3 \mathrm{~d}$ electron excited into the $\sigma^{*}$ orbital, leads to an estimated Auger electron kinetic energy of $479.4 \mathrm{eV}$ for the $\mathrm{M}_{5} \mathrm{~N}_{45} \mathrm{~N}_{45}$ transitions. The resulting shift, based on our calculated ionization energies, is $8.8 \mathrm{eV}$, compared to the experimental value of $7.6 \mathrm{eV}$. Thus, our theoretical orbital ionization energies in $3 \mathrm{~d}$ excited and $3 \mathrm{~d}$ ionized $\mathrm{CH}_{3} \mathrm{I}$ provide a reasonable estimation of the shift, although the estimated kinetic energies are lower than the experimental values by $\sim 25 \mathrm{eV}$. The same procedure for the $\mathrm{M}_{5} \mathrm{~N}_{23} \mathrm{~N}_{45}$ and $\mathrm{M}_{5} \mathrm{~N}_{1} \mathrm{~N}_{45}$ transitions results in estimated shifts of 8.7 and $8.8 \mathrm{eV}$, respectively. Hence, our theoretically estimated shifts for the $\mathrm{M}_{5} \mathrm{~N}_{45} \mathrm{~N}_{45}, \mathrm{M}_{5} \mathrm{~N}_{23} \mathrm{~N}_{45}$, and $\mathrm{M}_{5} \mathrm{~N}_{1} \mathrm{~N}_{45}$ transitions are about the same. For the $\mathrm{M}_{5} \mathrm{~N}_{45} \mathrm{O}_{23}$ transition, our estimated shift $(8.2 \mathrm{eV})$ is smaller, in accord with the smaller observed shift $(6.9 \mathrm{eV})$. Therefore, the predicted and measured shifts in the Auger electron kinetic energies appear to follow the same trends.

\section{Auger electron angular distributions}

The theoretical investigation carried out by Berezhko et al. ${ }^{28}$ shows that, in the non-relativistic limit, the align- 
ment resulting from inner shell photoionization of an atomic $\mathrm{d}$ orbital can be expressed as ${ }^{34}$

$$
\begin{aligned}
A_{20}\left(\mathrm{D}_{5 / 2}\right) & =\frac{-2}{5} \sqrt{\frac{2}{7}} \frac{\left(1+\frac{7}{2} \gamma\right)}{(1+\gamma)}, \\
A_{20}\left(\mathrm{D}_{3 / 2}\right) & =\frac{-1}{5} \frac{\left(1+\frac{7}{2} \gamma\right)}{(1+\gamma)}, \\
\gamma & =\frac{2}{3} \frac{\mathrm{R}_{3 \mathrm{~d} \rightarrow \varepsilon \mathrm{p}}^{2}}{\mathrm{R}_{3 \mathrm{~d} \rightarrow \varepsilon \mathrm{f}}^{2}}
\end{aligned}
$$

where $\mathrm{R}_{3 \mathrm{~d} \rightarrow \varepsilon \mathrm{p}}$ and $\mathrm{R}_{3 \mathrm{~d} \rightarrow \varepsilon \mathrm{f}}$ are the radial dipole matrix elements for the photoionization of the $3 \mathrm{~d}$ electron into the $\mathrm{p}$ and $\mathrm{f}$ continua, respectively.

For the non-spherical potential of a molecule, a considerably larger number of partial waves are expected to contribute to the cross section and angular distribution. Figure 8(a) shows the photoionization cross section for $\mathrm{CH}_{3} \mathrm{I}$, calculated using the methods described previously, ${ }^{72}$ along with the theoretical partial wave composition of the photoelectron obtained by expanding around the I atom. ${ }^{49}$ Apart from the region near threshold, Fig. 8(a) shows that the $l=3$ partial wave dominates the total I $3 \mathrm{~d}$ photoionization cross section. Thus, the photoionization process appears to be essentially atomic-like. In general, the $l=3$ cross section is expected to be much larger than the $l=1$ cross section, except close to threshold where the $l=3$ component is suppressed by the centrifugal barrier in the effective potential. ${ }^{47}$

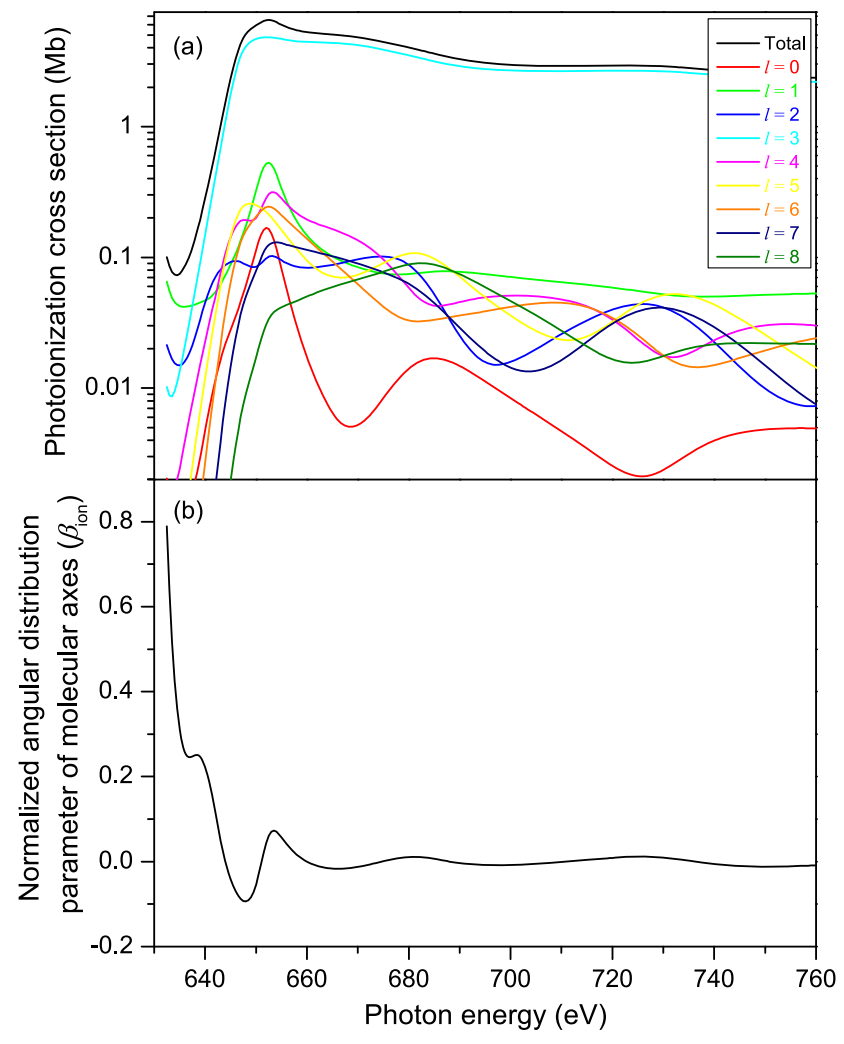

FIG. 8. (a) The calculated (CMS - X $\alpha$ ) I 3d photoionization cross section of $\mathrm{CH}_{3} \mathrm{I}$ decomposed into $l$-wave components and expanded around the I atom. (b) The calculated, normalized, angular distribution parameter $\left(\beta_{\text {ion }}\right)$ characterizing the spatial distribution of molecular axes in $\mathrm{CH}_{3} \mathrm{I}$ in the $\mathrm{I} 3 \mathrm{~d}^{-1}$ ionized state.
Using the same photoionization calculation, the spatial distribution of molecular axes, $I(\theta)$, in the $\mathrm{I} 3 \mathrm{~d}^{-1}$ ionized state can be evaluated from a rotation of the molecular frame dipole matrix elements into the lab frame, and integration over all azimuthal orientation and all electron emission directions. The alignment of molecular axes can be expressed as a distribution having the form ${ }^{73}$

$$
I(\theta)=1+\beta_{\text {ion }} P_{2}\left(\cos \theta_{\text {ion }}\right),
$$

where $\beta_{\text {ion }}$ is the normalized angular distribution parameter, $P_{2}\left(\cos \theta_{\text {ion }}\right)$ is the Legendre polynomial of second order, and $\theta_{\text {ion }}$ is the angle between the polarization axis of the plane polarized radiation and the molecular axis (the $\mathrm{C}-\mathrm{I}$ axis in $\mathrm{CH}_{3} \mathrm{I}$ ). The results of our calculation, plotted in Fig. 8(b), show that, apart from in a small energy range close to threshold, the molecular axes in the ionized state are not aligned to any significant extent.

In our analysis of the Auger electron angular distributions, following excitation or ionization of the I $3 \mathrm{~d}$ electron in $\mathrm{CH}_{3} \mathrm{I}$, we assume that the process can be considered as an atomic phenomenon and that Eqs. (5)-(7) are applicable. This assumption is supported by (i) the observation that the $3 \mathrm{~d}$ electron density is localized on the I atom, and well isolated from the delocalized molecular orbitals, and (ii) the calculated negligible molecular axis alignment in the ionized state, apart from close to threshold. The dominance of the $l=3$ component in the photon energy range encompassed by the present measurements results in $\gamma \rightarrow 0$. In this limit, ${ }^{28}$

$$
A_{20}\left(\mathrm{D}_{5 / 2}\right)=-0.21 \quad \text { and } \quad A_{20}\left(\mathrm{D}_{3 / 2}\right)=-0.20,
$$

where these values of the alignment parameter refer to plane polarized radiation with the quantization axis parallel to the electric vector of the radiation. Our convention is different to that used in the earlier theoretical work and requires that the values of $A_{20}$ reported by Berezhko et al. ${ }^{28}$ be multiplied by -2 to be compared with the present values. Berezhko et al. ${ }^{28}$ show that much larger values of the alignment parameter may be reached in the vicinity of the ionization threshold. In the limit $\gamma \rightarrow \infty, A_{20}\left(\mathrm{D}_{5 / 2}\right)=-0.75$ and $A_{20}\left(\mathrm{D}_{3 / 2}\right)=-0.70$. The values of the alignment parameter given in Eq. (9) have been used to evaluate the Auger electron intrinsic anisotropy parameter $\left(\alpha_{2}\right)$, following I $3 \mathrm{~d}$ photoionization, using Eq. (2), where the $\beta$ parameter is obtained from our fitting of the Auger electron spectra. Berezhko et al. ${ }^{28}$ have also shown that the degree of alignment due to a vacancy in the $3 \mathrm{~d}_{5 / 2}$ subshell of xenon reaches a fairly constant value once the energy is more than a few $\mathrm{eV}$ above the ionization threshold. The alignment due to a vacancy in the $3 d_{3 / 2}$ subshell would be expected to exhibit a similar energy dependence. Thus, the use of the limiting values of $A_{20}$, given in Eq. (9), for the evaluation of the I $3 \mathrm{~d}$ Auger electron intrinsic anisotropy parameters appears reasonable.

For photoexcitation by plane polarized radiation from an atomic ground state having $J=0$ to a state having $J=1$, $A_{20}$ has an energy independent value of $-\sqrt{2} .^{41}$ Ignoring the effects of the rotational structure of the molecule, we can use this atomic value to obtain the $\alpha_{2}$ parameters from the 
$\beta$ parameters extracted from our resonantly excited Auger spectra recorded at photon energies of 620.4 and $632.0 \mathrm{eV}$.

Figure 9 shows the $\beta$ parameters derived from our normal Auger spectra for the $\mathrm{M}_{45} \mathrm{~N}_{45} \mathrm{~N}_{45}$ transitions. It is evident that, for a particular final state, the values, in general, show little variation with photon energy. However, a few of the values obtained from the spectra recorded at $646.0 \mathrm{eV}$ depart from those recorded at the other photon energies. This discrepancy, particularly for the $\mathrm{M}_{4}$ Auger peaks, may be due to the small I $3 \mathrm{~d}_{3 / 2}$ ionization cross section near threshold. ${ }^{49}$ The low cross section results in a low Auger electron yield and hence in a less reliable fit to the associated spectra. The observed independence of the Auger electron $\beta$ parameters on photon energy is consistent with the expectation that the alignment does not vary significantly across the photon energy range over which these measurements were taken. Our values for the $\alpha_{2}$ parameters, obtained by averaging the $\beta$ parameters for a specific final state over several photon energies, and using $A_{20}\left(\mathrm{D}_{5 / 2}\right)=-0.21$ and $A_{20}\left(\mathrm{D}_{3 / 2}\right)=-0.20$, are listed in Table I. The theoretical $\alpha_{2}$ parameters for the $\mathrm{M}_{45} \mathrm{~N}_{45} \mathrm{~N}_{45}$ transitions in xenon, calculated by $\mathrm{Chen}^{42}$ using the multiconfiguration Dirac-Fock model, are also given in Table I. Although we are unable to resolve all the final states, the overall agreement between our results for the iodine $\mathrm{M}_{45} \mathrm{~N}_{45} \mathrm{~N}_{45}$ transitions in $\mathrm{CH}_{3} \mathrm{I}$ and those predicted for xenon is good.

This good overall agreement is not unexpected because the initial and final states involved in the $\mathrm{M}_{45} \mathrm{~N}_{45} \mathrm{~N}_{45}$ transitions in $\mathrm{Xe}$ are essentially the same as those involved in the corresponding transitions in $\mathrm{CH}_{3} \mathrm{I}$. The Auger decay matrix elements contain an energy dependence associated with the Auger electron kinetic energy. However, for a particular transition, the Auger electron kinetic energy in xenon is similar to that in $\mathrm{CH}_{3} \mathrm{I}$. Hence, this energy dependence may not significantly affect this comparison.

Our results are in reasonable accord with those obtained by Karvonen et al. ${ }^{29}$ for the normal $\mathrm{M}_{45} \mathrm{~N}_{45} \mathrm{~N}_{45}$ spectrum in xenon, although there are some significant discrepancies.

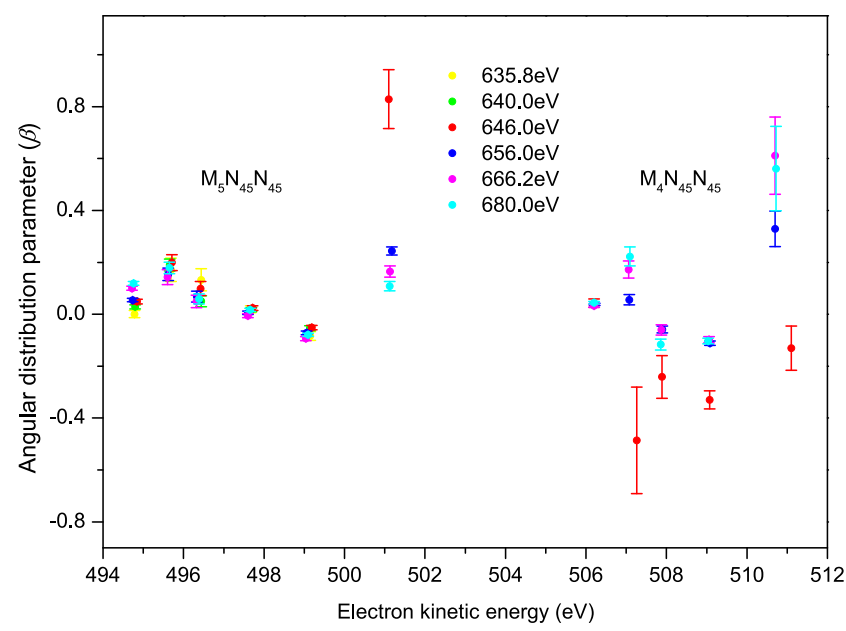

FIG. 9. The angular distribution parameters for the $\mathrm{M}_{45} \mathrm{~N}_{45} \mathrm{~N}_{45}$ transitions in $\mathrm{CH}_{3} \mathrm{I}$ derived from the normal Auger spectra recorded with vertically and horizontally polarized radiation.
For transitions leading to a two-hole final state with total angular momentum $J=0$, the Auger electron is characterized by a single partial wave, irrespective of the coupling scheme. ${ }^{34}$ Under these conditions, $\alpha_{2}=-1$ for the $\mathrm{M}_{4} \mathrm{~N}_{45} \mathrm{~N}_{45}{ }^{1} \mathrm{~S}_{0}$ and ${ }^{3} \mathrm{P}_{0}$ states and $\alpha_{2}=-1.069$ for the $\mathrm{M}_{5} \mathrm{~N}_{45} \mathrm{~N}_{45}{ }^{1} \mathrm{~S}_{0}$ and ${ }^{3} \mathrm{P}_{0}$ states. Using our $\beta$ value of $0.172 \pm 0.011$ for the $\mathrm{M}_{4} \mathrm{~N}_{45} \mathrm{~N}_{45}$ ${ }^{1} \mathrm{~S}_{0}$ state, together with the theoretically predicted $\alpha_{2}$ value of -1.0 , results in $A_{20}=-0.172 \pm 0.011$, which is in reasonable accord with our assumed theoretical value of -0.20 . Our $\beta$ value for the $\mathrm{M}_{5} \mathrm{~N}_{45} \mathrm{~N}_{45}{ }^{1} \mathrm{~S}_{0}$ state results in a value of $A_{20}$ which is in slightly poorer agreement with the theoretical prediction.

For our $3 \mathrm{~d}_{3 / 2,5 / 2} \rightarrow \sigma^{*}$ resonantly excited Auger spectra, there are no experimental or theoretical $\alpha_{2}$ parameters with which to compare our results (Table II). In general, the Auger electron angular distributions following resonant excitation are more anisotropic than those associated with non-resonant ionization. This reflects the higher degree of alignment induced in the photoexcited neutral state.

Figure 10 shows the $\beta$ parameters for the five peaks used to fit the $\mathrm{M}_{4} \mathrm{~N}_{45} \mathrm{O}_{23}$ and the $\mathrm{M}_{5} \mathrm{~N}_{45} \mathrm{O}_{23}$ transitions. Again, unfortunately, there are no theoretically predicted $\alpha_{2}$ parameters to help assess our results.

Theoretical values for the $\alpha_{2}$ parameters associated with the $\mathrm{M}_{4} \mathrm{~N}_{23} \mathrm{~N}_{45}{ }^{42}$ and the $\mathrm{M}_{5} \mathrm{~N}_{23} \mathrm{~N}_{45}{ }^{42,44}$ transitions in xenon have been reported, and the results from these two calculations are in good agreement with each other. However, it is not straight forward correlating these predictions with the experimental data shown in Fig. 5. The experimental results indicate that the $\beta$ parameter is slightly positive throughout most of the electron kinetic energy range covered by the $\mathrm{M}_{45} \mathrm{~N}_{23} \mathrm{~N}_{45}$ transitions except in the two regions containing the doublets. In contrast, the theoretical values for xenon exhibit a marked dependence on the final state.

Similar comments can be made for the $\beta$ parameter associated with the $\mathrm{M}_{45} \mathrm{~N}_{1} \mathrm{~N}_{45}$ transitions in $\mathrm{CH}_{3} \mathrm{I}$ (Fig. 6) for the resonantly excited spectrum recorded at a photon energy of $620.4 \mathrm{eV}$. The experimental data show that $\beta$ is close to zero

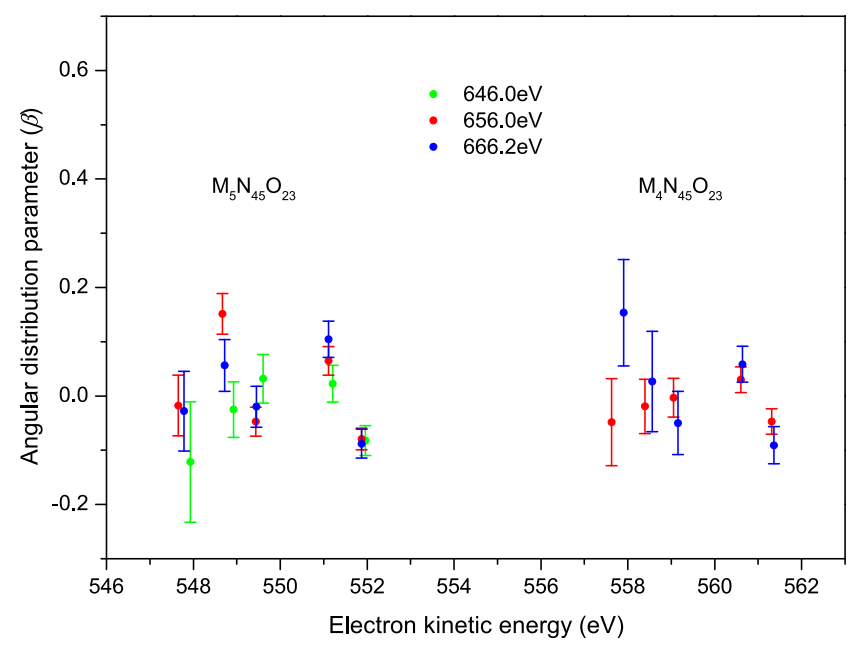

FIG. 10. The angular distribution parameters for the $\mathrm{M}_{45} \mathrm{~N}_{45} \mathrm{O}_{23}$ transitions in $\mathrm{CH}_{3} \mathrm{I}$ for the normal Auger spectra recorded at photon energies of $646.0 \mathrm{eV}$ (green), $656.0 \mathrm{eV}$ (red), and $666.2 \mathrm{eV}$ (blue) with vertically and horizontally polarized radiation. 
across the entire band structure whereas the theoretical predictions for xenon, although in good agreement with each other, predict widely ranging values. ${ }^{42,44}$

\section{SUMMARY}

Synchrotron radiation has been employed to record the normal and resonantly excited Auger electron spectra associated with the $\mathrm{I} 3 \mathrm{~d}$ level in $\mathrm{CH}_{3} \mathrm{I}$. These spectra have been measured with horizontally and vertically plane polarized radiation, thereby allowing the $\beta$ parameters to be determined. In the photon energy range over which spectra were recorded, the I $3 \mathrm{~d}$ photoionization cross section in $\mathrm{CH}_{3} \mathrm{I}$ appears very atomic-like and is dominated by transitions into the $\varepsilon$ f continuum. Under these conditions, the theoretical value of the alignment parameter characterizing the intermediate, core ionized state of an atom remains constant, independent of the photon energy. This theoretical value has been used to obtain the Auger electron intrinsic anisotropy parameters from the measured $\beta$ parameters.

Our experimentally derived $\alpha_{2}$ parameters have been compared with the corresponding predictions for Xe 3d. For the $\mathrm{M}_{45} \mathrm{~N}_{45} \mathrm{~N}_{45}$ decay, the I 3d Auger spectra are very similar to those measured for $\mathrm{Xe} \mathrm{3d}$, and our $\alpha_{2}$ parameters are in accord with those calculated for the corresponding transitions in xenon. The Auger electron spectra associated with the I $3 \mathrm{~d}_{45} \mathrm{~N}_{23} \mathrm{~N}_{45}$ and $\mathrm{M}_{45} \mathrm{~N}_{1} \mathrm{~N}_{45}$ transitions generally resemble those for the corresponding transitions in xenon and molecular iodine, but some differences are apparent. Our experimental $\alpha_{2}$ parameters for these two transitions do not exhibit the marked dependence on the final ionic state that is predicted in xenon. The Auger electron spectrum due to the I $3 \mathrm{~d} \mathrm{M}_{45} \mathrm{~N}_{45} \mathrm{O}_{23}$ transitions shows little molecular character and resembles that due to the corresponding transitions in xenon. This is consistent with our calculations which show that the outermost $2 \mathrm{e}$ orbital retains a strong iodine $5 \mathrm{p}$ lone-pair character. The Auger electron kinetic energy associated with a specific transition in the normal spectrum is shifted to higher energy in the resonantly excited spectrum. The observed shift has been verified using calculated transition energies for I $3 d$ excited and I $3 d$ ionized $\mathrm{CH}_{3} \mathrm{I}$.

\section{SUPPLEMENTARY MATERIAL}

See supplementary material for fits of the resonantly excited Auger electron spectra, orbital plots, and orbital ionization energies of I $3 \mathrm{~d}$ excited and $\mathrm{I} 3 \mathrm{~d}$ ionized $\mathrm{CH}_{3} \mathrm{I}$.

\section{ACKNOWLEDGMENTS}

D.M.P.H. thanks Kiyoshi Ueda (Tohoku University) and Stephen Southworth (Argonne National Laboratory) for helpful discussions. R.F. is grateful to the Engineering and Physical Sciences Research Council (EPSRC) for a research studentship. D.R. acknowledges support by the Chemical Sciences, Geosciences, and Biosciences Division, Office of Basic Energy Sciences, Office of Science, U.S. Department of Energy under Award No. DE-FG02-86ER13491. S.T.P. was supported by the U. S. Department of Energy, Office of
Science, Office of Basic Energy Sciences, Division of Chemical Sciences, Geosciences, and Biosciences under Contract No. DE-AC02-06CH11357. N.A.B. is grateful for support from the EPSRC [Grant No. EP/N002148/1]. D.M.P.H. is grateful to the Science and Technology Facilities Council (United Kingdom) for financial support. We are grateful to the SOLEIL staff for running the facility and providing beamtime under Project No. 20150786.

\section{APPENDIX: PEAK FITTING PROCEDURE FOR THE NORMAL AND RESONANT AUGER ELECTRON SPECTRA}

\section{Fitting the normal Auger spectrum}

The overall line shape associated with a normal (nonresonant) Auger transition arises from three contributions:

(i) The initial state level width that can be distorted by PCI between the photoelectron and the subsequently emitted Auger electron in the field of the positively charged ion. The effect of PCI broadening can be taken into account by implementing an approximate line shape in the analysis of the $\mathrm{CH}_{3} \mathrm{I} \mathrm{M}_{45}$ Auger electron peaks. The PCI broadening peak shape, $P^{\mathrm{PCI}}\left(E^{\mathrm{kin}}\right)$, can be expressed using an analytical formula given in the work of Armen et al., ${ }^{59}$

$$
\begin{aligned}
& P^{\mathrm{PCI}}\left(E^{\mathrm{kin}}, E^{0}, \Gamma, C\right) \\
&=\left(\frac{\Gamma}{2 \pi}\right)\left(\frac{\pi C}{\frac{1}{4} \Gamma^{2}+\left(E^{\mathrm{kin}}-E^{0}\right)^{2}}\right) \\
& \times\left(\frac{\exp \left[2 C \arctan \left(2\left(E^{\mathrm{kin}}-E^{0}\right)^{2} / \Gamma\right)\right]}{\sinh (\pi C)}\right),
\end{aligned}
$$

where $E^{\mathrm{kin}}, E^{0}$, and $\Gamma$ refer to the photoelectron kinetic energy, peak position, and core-hole width, respectively. $C$ is a fitting parameter which describes the distortion of the Lorentzian line shape and is dependent on the photoelectron excess energy.

(ii) The final state (symmetrical) level width, $\Gamma^{\mathrm{f}}$, that is represented by a Lorentzian profile, $L^{\text {Auger }}\left(E^{\mathrm{kin}}, \Gamma^{\mathrm{f}}\right)$,

$$
L^{\text {Auger }}\left(E^{\mathrm{kin}}, \Gamma^{\mathrm{f}}\right)=\left(\frac{1}{2 \pi}\right)\left(\frac{\Gamma^{\mathrm{f}}}{\left(\frac{\Gamma^{\mathrm{f}}}{2}\right)^{2}+\left(E^{\mathrm{kin}}-E^{0}\right)^{2}}\right) .
$$

(iii) The total instrumental broadening: a Gaussian due to the convolution of the Gaussian broadenings associated with the spectrometer (62.5 meV) and the Doppler contribution (23 meV). This corresponds to a Full Width at Half Maximum (FWHM) of the instrumental broadening of $66.6 \mathrm{meV}$. The photon resolution does not play a role and the associated peak shape is given by

$$
G\left(E^{\mathrm{kin}}, \sigma\right)=\frac{1}{\sigma \sqrt{2 \pi}} \exp \left[\frac{-\left(E^{\mathrm{kin}}\right)^{2}}{2 \sigma^{2}}\right] .
$$

The peak shape for a specific normal (non-resonant) Auger transition can therefore be expressed as 


$$
\begin{aligned}
P\left(E^{\mathrm{kin}}, \theta, \sigma, \Gamma^{\mathrm{f}}, \Gamma, C, E^{0}, W_{i \rightarrow f}^{\mathrm{T}}, \beta\right) & \\
= & \frac{W_{i \rightarrow f}^{\mathrm{T}}}{4 \pi}\left(1+\beta P_{2}(\cos \theta)\right) \times\left(P^{\mathrm{PCI}}\left(E^{\mathrm{kin}}, E^{0}, \Gamma, C\right)\right. \\
& \left.* L^{\text {Auger }}\left(E^{\mathrm{kin}}, \Gamma^{\mathrm{f}}\right) * G\left(E^{\mathrm{kin}}, \sigma\right)\right),
\end{aligned}
$$

where the amplitude of the peak is related to the angularly resolved differential cross section,

$$
\frac{d W_{J_{i} \rightarrow J_{f}}(\theta)}{d \omega}=\frac{W_{J_{i} \rightarrow J_{f}}^{T}}{4 \pi}\left[1+\beta P_{2}(\cos \theta)\right],
$$

where $W_{i \rightarrow f}^{\mathrm{T}}$ is the total Auger decay rate for a particular transition between intermediate and final ionic states, $\beta$ is the electron angular distribution parameter, $P_{2}(\cos \theta)$ is the Legendre polynomial of second order, and $\theta$ is the Auger electron ejection angle relative to the polarization axis of the plane polarized radiation.

The fitting function for a non-resonant Auger group, connected with a single core hole, is therefore

$$
\begin{aligned}
I_{\mathrm{M}_{i}} & \left(E^{\mathrm{kin}}, \theta, \sigma_{j}, \Gamma_{j}^{\mathrm{f}}, \Gamma_{\mathrm{M}_{i}}, C_{j}, E_{j}^{0}, W_{i \rightarrow f, j}^{\mathrm{T}}, \beta_{j}\right) \\
& =\sum_{j=1}^{j \max } P\left(E^{\mathrm{kin}}, \theta, \sigma_{j}, \Gamma_{j}^{\mathrm{f}}, \Gamma_{\mathrm{M}_{i}}, C_{j}, E_{j}^{0}, W_{i \rightarrow f, j}^{\mathrm{T}}, \beta_{j}\right) .
\end{aligned}
$$

Here $j$ is used to denote the peak number in an Auger group and $j^{\text {max }}$ denotes the total number of peaks within the group. $\mathbf{M}_{i}$ is used to indicate whether the intensity of a particular Auger group, connected with a particular intermediate state, is either a $\mathrm{M}_{5}\left(3 \mathrm{~d}_{5 / 2}{ }^{-1}\right)$ or a $\mathrm{M}_{4}\left(3 \mathrm{~d}_{3 / 2}{ }^{-1}\right)$ core hole.

The functional form used to fit the Auger spectra, at a given photon energy, can therefore be expressed as

$$
\begin{aligned}
& I\left(E^{\mathrm{kin}}, a, b, \theta, \sigma_{j, j^{\prime}}, \Gamma_{j, j^{\prime}}^{\mathrm{f}}, \Gamma_{\mathrm{M}_{i}}, C_{j, j^{\prime}}, E_{j, j^{\prime}}^{0}, W_{i \rightarrow f, j, j^{\prime}}^{\mathrm{T}}, \beta_{j, j^{\prime}}\right)=a+b E^{\mathrm{kin}}+\sum_{j=1}^{j^{\max }} I_{\mathrm{M}_{5}}\left(E^{\mathrm{kin}}, \theta, \sigma_{j}, \Gamma_{j}^{\mathrm{f}}, \Gamma_{\mathrm{M}_{5}}, C_{j}, E_{j}^{0}, W_{i \rightarrow f, j}^{\mathrm{T}}, \beta_{j}\right) \\
& +\sum_{j^{\prime}=1}^{j^{\prime \max }} I_{\mathrm{M}_{4}}\left(E^{\mathrm{kin}}, \theta, \sigma_{j^{\prime}}, \Gamma_{j^{\prime}}^{\mathrm{f}}, \Gamma_{\mathrm{M}_{4}}, C_{j^{\prime}}, E_{j^{\prime}}^{0}, W_{i \rightarrow f, j^{\prime}}^{\mathrm{T}}, \beta_{j^{\prime}}\right)
\end{aligned}
$$

The double indices of $j$ and $j^{\prime}$ are used to denote the number of peaks in the $\mathrm{M}_{5}$ and $\mathrm{M}_{4}$ Auger groups, respectively. The coefficients $a$ and $b$ are used to describe a linear energy dependent background, which can be used to remove superfluous counts not associated with the Auger decay process.

The peak intensity, for the total spectrum at a particular photon energy, was fitted to the spectra recorded with vertically or horizontally plane polarized radiation using $\theta=0^{\circ}$ or $\theta=90^{\circ}$, respectively. The global minimization function was then constructed from the two separate minimization functions, associated with each of the vertically and horizontally polarized spectra.

In the fitting routine, both of the widths in the peak shape (associated with the total instrumental broadening and the final state broadening) were constrained to be equivalent for all peaks regardless of the intermediate core-hole state and for both polarization states such that $\sigma_{j}=\cdots=\sigma_{j^{\max }}$ and $\Gamma_{j}^{\mathrm{f}}=\cdots=\Gamma_{j^{\mathrm{max}}}^{\mathrm{f}}$ for $\theta=0^{\circ}$ and $\theta=90^{\circ}$.

Bounds were placed on the fitting parameters to ensure that they had physically significant values. For example, the widths, peak intensities, and peak positions were constrained to be positive, and the $\beta$ parameter value was constrained to lie between -1 and 2 .

Finally, we note that the width associated with the total instrumental broadening and that associated with the intermediate state core-hole broadening were not treated as fitting parameters but were held fixed during the weighted least squares fitting routine. The exact values used were based on the extracted width from fits of the $3 d_{5 / 2}$ and $3 d_{3 / 2}$ photolines and from estimates of the beamline resolution.

\section{Fitting the resonant Auger spectrum}

For resonant Auger transitions, if the photon bandwidth is narrower than the level width of the neutral excited state $\left(3 d_{5 / 2}{ }^{-1} \sigma^{*}\right.$ or $3 d_{3 / 2}{ }^{-1} \sigma^{*}$ in our case), then the initial state width can be neglected and replaced by a Gaussian representing the photon bandwidth. ${ }^{7}$ As a resonantly excited state is being prepared and there is no photoelectron produced during initial photoexcitation, the effects of PCI are not considered here.

The total line shape for a specific Auger transition is therefore given by the convolution of two contributions:

(i) The total instrumental broadening: a Gaussian due to the convolution of the Gaussian broadenings associated with the spectrometer $(62.5 \mathrm{meV})$, the Doppler contribution $(23 \mathrm{meV})$, and the photon bandwidth $(\sim 300 \mathrm{meV})$.

(ii) The final state $\left(4 \mathrm{~d}^{-2} \sigma^{*}\right)$ level width that is represented by a Lorentzian profile. The total line shape used in our fitting is based on the notes described in Ref. 58. Since the line shape is given by a convolution of a Gaussian profile $G(x, \sigma)$ and a Lorentzian profile $L\left(x, \gamma^{\mathrm{f}}\right)$, we simply use a Voigt line shape $V\left(x, \sigma, \gamma^{\mathrm{f}}\right)$ in our fitting routine, 


$$
\begin{aligned}
& V\left(x, \sigma, \gamma^{\mathrm{f}}\right)=\int_{-\infty}^{\infty} G\left(x^{\prime}, \sigma\right) L\left(x-x^{\prime}, \gamma^{\mathrm{f}}\right) d x^{\prime}, \\
& G(x, \sigma)=\frac{1}{\sigma \sqrt{2 \pi}} \exp \left[\frac{-x^{2}}{2 \sigma^{2}}\right], \\
& L\left(x, \gamma^{\mathrm{f}}\right)=\frac{\gamma^{\mathrm{f}} / \pi}{\left(\gamma^{\mathrm{f}}\right)^{2}+x^{2}} .
\end{aligned}
$$

Here $\gamma^{\mathrm{f}}$ is the Half Width at Half Maximum (HWHM) of the Lorentzian profile connected with the final state level width and $\sigma$ is the standard deviation of the Gaussian profile related to the total instrumental broadening. The HWHM, $\alpha$, is $155 \mathrm{meV}$ and is related to $\sigma$ by $\alpha=\sigma \sqrt{2 \ln 2}$. In terms of electron kinetic energy, $E^{\mathrm{kin}}, x=E^{\mathrm{kin}}-E^{0}$, where $E^{0}$ is the central kinetic energy of an Auger peak. There is no closed form for the Voigt profile, but it is related to the real part of the Faddeeva function, $w(z)$, by

$$
\begin{gathered}
V\left(x, \sigma, \gamma^{\mathrm{f}}\right)=\frac{\operatorname{Re}[w(z)]}{\sigma \sqrt{2 \pi}}, \\
z=\frac{x+i \gamma^{\mathrm{f}}}{\sigma \sqrt{2}} .
\end{gathered}
$$

The peak shape for a specific resonant Auger transition can therefore be expressed as

$$
\begin{aligned}
P^{\operatorname{Res}} & \left(E^{\mathrm{kin}}, \theta, \sigma, \gamma^{\mathrm{f}}, E^{0}, W_{i \rightarrow f}^{\mathrm{T}}, \beta\right) \\
& =\frac{W_{i \rightarrow f}^{\mathrm{T}}}{4 \pi}\left(1+\beta P_{2}(\cos \theta)\right) \times V\left(E^{\mathrm{kin}}, E^{0}, \sigma, \gamma^{\mathrm{f}}\right),
\end{aligned}
$$

where the amplitude of the peak is again related to the angularly resolved differential cross section. The total fitting function for a given resonant Auger group, containing multiple peaks and terms associated with background electron intensity, is given by

$$
\begin{aligned}
& I\left(E^{\mathrm{kin}}, a, b, \theta, \sigma_{k}, \gamma_{k}^{\mathrm{f}}, E_{k}^{0}, W_{i \rightarrow f, k}^{\mathrm{T}}, \beta_{k}\right) \\
& \quad=a+b E^{\mathrm{kin}}+\sum_{k=1}^{k^{\mathrm{max}}} P^{\operatorname{Res}}\left(E^{\mathrm{kin}}, \theta, \sigma_{k}, \gamma_{k}^{\mathrm{f}}, E_{k}^{0}, W_{i \rightarrow f, k}^{\mathrm{T}}, \beta_{k}\right) .
\end{aligned}
$$

Here $k$ is used to denote the peak number in a particular resonant Auger group and $k^{\max }$ denotes the total number of peaks within the group. At a given photon energy, the above function form was fitted to the spectra recorded with vertically or horizontally plane polarized radiation, simultaneously. This procedure made use of a global minimization function constructed, in a manner similar to that described in relation to the normal Auger spectra, from the individual functions associated with the vertically and horizontally polarized spectra. In addition, bounds were placed on the fitting parameters to ensure that they had physically significant values and the width associated with the total instrumental broadening was held fixed during the fitting routine.

${ }^{1}$ K. Siegbahn, C. Nordling, G. Johansson, J. Hedman, P. F. Heden, K. Hamrin, U. Gelius, T. Bergmark, L. O. Werme, R. Manne, and Y. Baer, ESCA Applied to Free Molecules (North Holland, Amsterdam, 1969).

${ }^{2}$ T. A. Carlson, Photoelectron and Auger Spectroscopy (Plenum Press, New York, 1975).

${ }^{3}$ H. Aksela, S. Aksela, and N. Kabachnik, in VUV and Soft X-Ray Photoionization, edited by U. Becker and D. A. Shirley (Plenum Press, New York, 1996), p. 401.
${ }^{4}$ V. Schmidt, Electron Spectrometry of Atoms using Synchrotron Radiation (Cambridge University Press, Cambridge, 1997).

${ }^{5}$ G. B. Armen, H. Aksela, T. Åberg, and S. Aksela, J. Phys. B: At., Mol. Opt. Phys. 33, R49 (2000).

${ }^{6}$ M. N. Piancastelli, J. Electron Spectrosc. Relat. Phenom. 107, 1 (2000).

${ }^{7}$ K. Ueda, J. Phys. B: At., Mol. Opt. Phys. 36, R1 (2003).

${ }^{8}$ S. Svensson, J. Phys. B: At., Mol. Opt. Phys. 38, S821 (2005).

${ }^{9}$ P. Morin and I. Nenner, Phys. Rev. Lett. 56, 1913 (1986).

${ }^{10} \mathrm{P}$. Morin and I. Nenner, Phys. Scr. T17, 171 (1987).

${ }^{11}$ H. Aksela, S. Aksela, M. Ala-Korpela, O.-P. Sairanen, M. Hotokka, G. M. Bancroft, K. H. Tan, and J. Tulkki, Phys. Rev. A 41, 6000 (1990).

${ }^{12}$ A. Menzel, B. Langer, J. Viefhaus, S. B. Whitfield, and U. Becker, Chem. Phys. Lett. 258, 265 (1996).

${ }^{13}$ E. Kukk, H. Aksela, O.-P. Sairanen, S. Aksela, A. Kivimäki, E. Nõmmiste, A. Ausmees, A. Kikas, S. J. Osborne, and S. Svensson, J. Chem. Phys. 104, 4475 (1996).

${ }^{14}$ E. Kukk, H. Aksela, O.-P. Sairanen, E. Nõmmiste, S. Aksela, S. J. Osborne, A. Ausmees, and S. Svensson, Phys. Rev. A 54, 2121 (1996).

${ }^{15}$ O. Björneholm, S. Sundin, S. Svensson, R. R. T. Marinho, A. Naves de Brito, F. Gel'mukhanov, and H. Ågren, Phys. Rev. Lett. 79, 3150 (1997).

${ }^{16}$ A. Kivimäki, E. Kukk, J. Karvonen, J. Mursu, E. Nõmmiste, H. Aksela, and S. Aksela, Phys. Rev. A 57, 2724 (1998).

${ }^{17}$ N. M. Kabachnik, K. Ueda, Y. Muramatsu, and Y. Sato, J. Phys. B: At., Mol. Opt. Phys. 31, 4791 (1998).

${ }^{18}$ E. Sokell, A. A. Wills, M. Wiedenhoeft, X. Feng, D. Rolles, and N. Berrah, J. Phys. B: At., Mol. Opt. Phys. 38, 1535 (2005).

${ }^{19}$ H. Sann, T. Havermeier, C. Müller, H.-K. Kim, F. Trinter, M. Waitz, J. Voigtsberger, F. Sturm, T. Bauer, R. Wallauer, D. Schneider, M. Weller, C. Goihl, J. Tross, K. Cole, J. Wu, M. S. Schöffler, H. Schmidt-Böcking, T. Jahnke, M. Simon, and R. Dörner, Phys. Rev. Lett. 117, 243002 (2016).

${ }^{20} \mathrm{P}$. Morin and C. Miron, J. Electron Spectrosc. Relat. Phenom. 185, 259 (2012).

${ }^{21}$ E. Antonsson, M. Patanen, C. Nicolas, S. Benkoula, J. J. Neville, V. L. Sukhorukov, J. D. Bozek, Ph. V. Demekhin, and C. Miron, Phys. Rev. A 92, 042506 (2015).

${ }^{22}$ D. Dill, J. R. Swanson, S. Wallace, and J. L. Dehmer, Phys. Rev. Lett. 45, 1393 (1980).

${ }^{23}$ B. Cleff and W. Mehlhorn, Phys. Lett. A 37, 3 (1971).

${ }^{24}$ S. Flügge, W. Mehlhorn, and V. Schmidt, Phys. Rev. Lett. 29, 7 (1972).

${ }^{25}$ W. Mehlhorn, in Atomic Inner-Shell Physics, edited by B. Crasemann (Plenum Press, New York, 1985), p. 119.

${ }^{26}$ C. N. Yang, Phys. Rev. 74, 764 (1948).

${ }^{27}$ E. G. Berezhko and N. M. Kabachnik, J. Phys. B: At. Mol. Phys. 10, 2467 (1977).

${ }^{28}$ E. G. Berezhko, N. M. Kabachnik, and V. S. Rostovsky, J. Phys. B: At. Mol. Phys. 11, 1749 (1978).

${ }^{29}$ J. Karvonen, A. Kivimäki, H. Aksela, S. Aksela, R. Camilloni, L. Avaldi, M. Coreno, M. de Simone, and K. C. Prince, Phys. Rev. A 59, 315 (1999).

${ }^{30}$ S. H. Southworth, P. H. Kobrin, C. M. Truesdale, D. Lindle, S. Owaki, and D. A. Shirley, Phys. Rev. A 24, 2257 (1981).

${ }^{31}$ S. Southworth, U. Becker, C. M. Truesdale, P. H. Kobrin, D. W. Lindle, S. Owaki, and D. A. Shirley, Phys. Rev. A 28, 261 (1983).

${ }^{32}$ T. A. Carlson, D. R. Mullins, C. E. Beall, B. W. Yates, J. W. Taylor, D. W. Lindle, B. P. Pullin, and F. A. Grimm, Phys. Rev. Lett. 60, 1382 (1988).

${ }^{33}$ T. A. Carlson, D. R. Mullins, C. E. Beall, B. W. Yates, J. W. Taylor, D. W. Lindle, and F. Grimm, Phys. Rev. A 39, 1170 (1989).

${ }^{34}$ B. Kämmerling, V. Schmidt, W. Mehlhorn, W. B. Peatman, F. Schaefers, and T. Schroeter, J. Phys. B: At., Mol. Opt. Phys. 22, L597 (1989).

${ }^{35}$ B. Kämmerling, B. Krässig, and V. Schmidt, J. Phys. B: At., Mol. Opt. Phys. 23, 4487 (1990).

${ }^{36}$ R. Sankari, A. Kivimäki, M. Huttula, H. Aksela, S. Aksela, M. Coreno, G. Turri, R. Camilloni, M. de Simone, and K. C. Prince, Phys. Rev. A 63, 032715 (2001).

${ }^{37}$ A. De Fanis, N. Saito, M. Kitajima, Y. Shimizu, K. Okada, H. Tanaka, I. Koyano, and K. Ueda, J. Phys. B: At., Mol. Opt. Phys. 34, L377 (2001).

${ }^{38}$ M. Kitajima, M. Okamoto, M. Hoshino, H. Tanaka, S. Fritzsche, N. M. Kabachnik, I. P. Sazhina, Y. Shimizu, and K. Ueda, J. Phys. B: At., Mol. Opt. Phys. 35, 3327 (2002).

${ }^{39}$ N. M. Kabachnik, I. P. Sazhina, I. S. Lee, and O. V. Lee, J. Phys. B: At., Mol. Opt. Phys. 21, 3695 (1988).

${ }^{40}$ N. M. Kabachnik and I. P. Sazhina, J. Phys. B: At., Mol. Opt. Phys. 21, 267 (1988).

${ }^{41}$ U. Hergenhahn, N. M. Kabachnik, and B. Lohmann, J. Phys. B: At., Mol. Opt. Phys. 24, 4759 (1991). 
${ }^{42}$ M. H. Chen, Phys. Rev. A 45, 1684 (1992).

${ }^{43}$ S. Fritzsche, Phys. Lett. A 180, 262 (1993).

${ }^{44}$ J. Tulkki, N. M. Kabachnik, and H. Aksela, Phys. Rev. A 48, 1277 (1993).

${ }^{45}$ J. Tukki, H. Aksela, and N. M. Kabachnik, Phys. Rev. A 48, 2957 (1993).

${ }^{46}$ A. Yu. Elizarov and I. I. Tupitsyn, J. Exp. Theor. Phys. 97, 658 (2003).

${ }^{47}$ S. T. Manson and J. W. Cooper, Phys. Rev. 165, 126 (1968).

${ }^{48}$ J. W. Cooper, Phys. Rev. 128, 681 (1962).

${ }^{49}$ R. Forbes, A. De Fanis, C. Bomme, D. Rolles, S. T. Pratt, I. Powis, N. A. Besley, M. Simon, S. Nandi, A. R. Milosavljević, C. Nicolas, J. D. Bozek, J. G. Underwood, and D. M. P. Holland, "Photoionization of the iodine 3d, $4 s$ and $4 p$ orbitals in methyl iodide," J. Chem. Phys. (submitted).

${ }^{50}$ J. Söderström, A. Lindblad, A. N. Grum-Grzhimailo, O. Travnikova, C. Nicolas, S. Svensson, and C. Miron, New J. Phys. 13, 073014 (2011).

${ }^{51}$ I. Powis, D. M. P. Holland, E. Antonsson, M. Patanen, C. Nicolas, C. Miron, M. Schneider, D. Yu. Soshnikov, A. Dreuw, and A. B. Trofimov, J. Chem. Phys. 143, 144304 (2015).

${ }^{52}$ H. Petersen, Opt. Commun. 40, 402 (1982).

${ }^{53}$ P. Baltzer, L. Karlsson, M. Lundqvist, and B. Wannberg, Rev. Sci. Instrum. 64, 2179 (1993).

${ }^{54}$ S. H. Southworth, A. C. Parr, J. E. Hardis, J. L. Dehmer, and D. M. P. Holland, Nucl. Instrum. Methods Phys. Res., Sect. A 246, 782 (1986).

${ }^{55}$ A. Kivimäki, U. Hergenhahn, B. Kempgens, R. Hentges, M. N. Piancastelli, K. Maier, A. Rüdel, J. J. Tulkki, and A. M. Bradshaw, Phys. Rev. A 63, $012716(2000)$

${ }^{56}$ L. O. Werme, T. Bergmark, and K. Siegbahn, Phys. Scr. 6, 141 (1972).

${ }^{57}$ J. Jauhiainen, A. Ausmees, A. Kivimäki, S. J. Osborne, A. Naves de Brito, S. Aksela, S. Svensson, and H. Aksela, J. Electron Spectrosc. Relat. Phenom. 69, 181 (1994).
${ }^{58}$ E. Jones et al., SciPy: Open Source Scientific Tools for Python, 2001, http://www.scipy.org.

${ }^{59}$ G. B. Armen, J. Tulkki, T. Åberg, and B. Crasemann, Phys. Rev. A 36, 5606 (1987).

${ }^{60}$ S. Aksela, H. Aksela, and T. D. Thomas, Phys. Rev. A 19, 721 (1979).

${ }^{61}$ H. Pulkkinen, H. Aksela, and S. Aksela, Phys. Rev. A 34, 1195 (1986).

${ }^{62}$ M. J. Frisch et al., GAussian 09, Revision D.01, Gaussian, Inc., Wallingford, CT, 2013.

${ }^{63}$ L. Karlsson, R. Jadrny, L. Mattsson, F. T. Chau, and K. Siegbahn, Phys. Scr. 16, 225 (1977).

${ }^{64}$ D. M. P. Holland, I. Powis, G. Öhrwall, L. Karlsson, and W. von Niessen, Chem. Phys. 326, 535 (2006).

${ }^{65}$ M. Pernpointner, J. P. Zobel, E. Fasshauer, and A. N. Sil, Chem. Phys. 407, 39 (2012).

${ }^{66}$ S. Svensson, N. Mårtensson, E. Basilier, P. A.. Malmquist, U. Gelius, and K. Siegbahn, Phys. Scr. 14, 141 (1976).

${ }^{67}$ G. Wendin and M. Ohno, Phys. Scr. 14, 148 (1976).

${ }^{68}$ P. J. Stephens, F. J. Devlin, C. F. Chabalowski, and M. J. Frisch, J. Phys. Chem. 98, 11623 (1994).

${ }^{69}$ A. T. B. Gilbert, N. A. Besley, and P. M. W. Gill, J. Phys. Chem. A 112, 13164 (2008).

${ }^{70}$ N. A. Besley, A. T. B. Gilbert, and P. M. W. Gill, J. Chem. Phys. 130, 124308 (2009).

${ }^{71}$ Y. Shao et al., Mol. Phys. 113, 184 (2015).

${ }^{72}$ I. Powis, Chem. Phys. 201, 189 (1995).

${ }^{73}$ S. M. Bellm, J. A. Davies, P. T. Whiteside, J. Guo, I. Powis, and K. L. Reid, J. Chem. Phys. 122, 224306 (2005). 